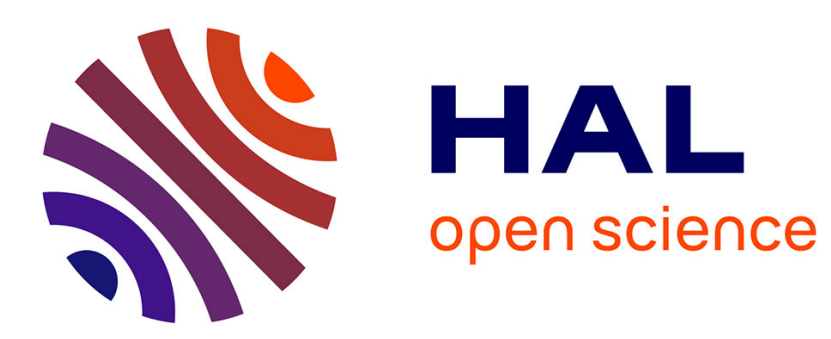

\title{
Persistent subplasma-frequency kinetic electrostatic electron nonlinear waves
}

T. W. Johnston, Y. Tyshetskiy, A. Ghizzo, Pierre Bertrand

\section{To cite this version:}

T. W. Johnston, Y. Tyshetskiy, A. Ghizzo, Pierre Bertrand. Persistent subplasma-frequency kinetic electrostatic electron nonlinear waves. Physics of Plasmas, 2009, 16 (4), pp.042105. 10.1063/1.3094061 . hal-01791837

\section{HAL Id: hal-01791837 https://hal.univ-lorraine.fr/hal-01791837}

Submitted on 14 May 2018

HAL is a multi-disciplinary open access archive for the deposit and dissemination of scientific research documents, whether they are published or not. The documents may come from teaching and research institutions in France or abroad, or from public or private research centers.
L'archive ouverte pluridisciplinaire HAL, est destinée au dépôt et à la diffusion de documents scientifiques de niveau recherche, publiés ou non, émanant des établissements d'enseignement et de recherche français ou étrangers, des laboratoires publics ou privés. 


\section{Persistent subplasma-frequency kinetic electrostatic electron nonlinear waves}

T. W. Johnston, Y. Tyshetskiy, A. Ghizzo, and P. Bertrand

Citation: Physics of Plasmas 16, 042105 (2009); doi: 10.1063/1.3094061

View online: https://doi.org/10.1063/1.3094061

View Table of Contents: http://aip.scitation.org/toc/php/16/4

Published by the American Institute of Physics

\section{Articles you may be interested in}

Electron holes in phase space: What they are and why they matter

Physics of Plasmas 24, 055601 (2017); 10.1063/1.4976854

On the nonlinear trapping nature of undamped, coherent structures in collisionless plasmas and its impact on stability

Physics of Plasmas 24, 032109 (2017); 10.1063/1.4978477

On the nature of kinetic electrostatic electron nonlinear (KEEN) waves

Physics of Plasmas 21, 034501 (2014); 10.1063/1.4868230

Longitudinal Ion Oscillations in a Hot Plasma

The Physics of Fluids 4, 139 (1961); 10.1063/1.1706174

Undamped electrostatic plasma waves

Physics of Plasmas 19, 092103 (2012); 10.1063/1.4751440

Hole equilibria in Vlasov-Poisson systems: A challenge to wave theories of ideal plasmas

Physics of Plasmas 7, 4831 (2000); 10.1063/1.1316767

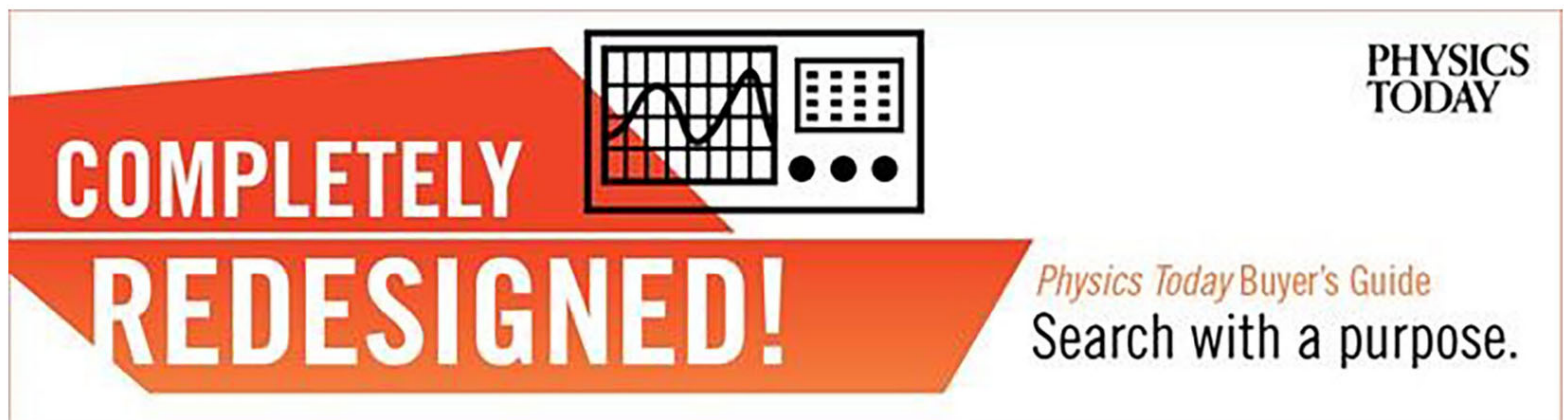




\title{
Persistent subplasma-frequency kinetic electrostatic electron nonlinear waves
}

\author{
T. W. Johnston, ${ }^{1}$ Y. Tyshetskiy, ${ }^{1,2}$ A. Ghizzo, ${ }^{3}$ and P. Bertrand ${ }^{3}$ \\ ${ }^{1}$ INRS-EMT, Varennes, Québec J3X 1S2, Canada \\ ${ }^{2}$ School of Physics, University of Sydney, New South Wales 2006, Australia \\ ${ }^{3}$ LPMIA Université Henri Poincaré, B.P. 239 1-54506 Nancy, cedex France
}

(Received 2 December 2008; accepted 12 February 2009; published online 10 April 2009)

\begin{abstract}
Driving a one-dimensional collisionless Maxwellian (Vlasov) plasma with a sufficiently strong longitudinal ponderomotive driver for a sufficiently long time results in a self-sustaining nonsinusoidal wave train with well-trapped electrons even for frequencies well below the plasma frequency, i.e., in the plasma wave spectral gap. Typical phase velocities of these waves are somewhat above the electron thermal velocity. This new nonlinear wave is being termed a kinetic electrostatic electron nonlinear (KEEN) wave. The drive duration must exceed the bounce period $\tau_{B}$ of the trapped electrons subject to the drive, as calculated from the drive force and the linear plasma response to the drive. For a given wavenumber a wide range of KEEN wave frequencies can be readily excited. The basic KEEN structure is essentially kinetic, with the trapped electron density variation being almost completely shielded by the free electrons, leaving just enough net charge to support the wave. () 2009 American Institute of Physics. [DOI: 10.1063/1.3094061]
\end{abstract}

\section{INTRODUCTION}

The original trigger for the activity reported here was the remarkable set of results obtained by Montgomery et al. ${ }^{1,2}$ in a series of laser-plasma interaction experiments. These experiments were aimed at improving our knowledge of stimulated Raman scattering (SRS) from EPWs from a single speckle of laser light and employed laser scattering as a key diagnostic. In addition to the expected EPW scattering signal, there was also an unexpected signal which was much smaller than that associated with SRS-related EPW $\left(\sim 10^{-3}\right.$ lower intensity) but nonetheless well out of the noise.

The extremely surprising aspect of this scattering result was that the frequency of the relatively weak electrostatic density wave associated with this signal was only a modest fraction $(\sim 0.37)$ of the plasma frequency, which put it right in the so-called frequency gap where no electrostatic wave ought to be, at least according to linear wave theory for a plasma with Maxwellian electrons. The observed wavevector was at a modest fraction (0.26) of the reciprocal Debye length, giving the electrostatic wave a phase velocity not far above the electron thermal velocity.

As indicated in the papers by Montgomery et al. ${ }^{1,2}$ and in a related analysis by Rose and Russell, ${ }^{3}$ waves having this character had been discussed previously in connection with in situ observations in the ionosphere and there termed EAWs for electron acoustic waves. ${ }^{4,5}$ Other analyses on waves of this kind created by imposition of initial values on the electron distribution function (but not cited by Montgomery et al.) had been published by Schamel ${ }^{6}$ and by Krapchev and Ram. ${ }^{7}$ Recently much more light has been shed on these scattering results ${ }^{1,2}$ by Strozzi et al. ${ }^{8}$ who dealt specifically with the problem of the generation of EAW-style phenomenon in the presence of strong SRS. (Their work will be discussed briefly at the end of this paper.)

The theory just cited $^{4-7}$ and work by many others ${ }^{9-16}$ (previous to the work by Strozzi et al. ${ }^{8}$ ) addressed the problem of the possible persistence of such EAW-style modes but only when imposed as initial states (with necessarily complicated structure in the electron velocity distribution function near the phase velocity). No obvious means exist to impose such complicated initial states other than by a simple act of will by theorists in a computer model. No discussion was given of how such a state could be generated in a uniform Maxwellian plasma in an actual experiment or in another plasma physics event. In view of experimental scattering results of Montgomery et al., the central question of interest is how a long wave train of electrostatic density modulations (as evidenced by the experimentally observed narrow scattering spectrum ${ }^{1,2}$ ) but at a frequency far below the plasma frequency can be obtained via some parametric resonance in spite of the unfavorable low-frequency linear response of a Maxwellian electron velocity distribution. (With no carefully crafted population of trapped electrons, this response is strongly damped and broad band.)

To explore the physics of such generation and to make contact with a possible laboratory experiment using the ponderomotive force (PF) between two opposing laser beams appropriately separated in frequency, it was decided (beginning in 2001) to undertake a study of the effects of applying a PF drive to a collisionless uniform Maxwellian plasma in an appropriate computer model for the one-dimensional (1D) electrostatic Vlasov electron plasma. This work was begun by Afeyan at Polymath Research and by Tudor Johnston at INRS-EMT.

In this paper we report the basic, least sophisticated concepts distilled from this simulation effort at INRS-EMT. The simulations demonstrated successful generation of indefinitely self-sustaining low-frequency waves, produced by using a PF drive which (as it turned out) needed to be applied for a limited time only. Because these waves are essentially 
kinetic and electrostatic, because they involve the electrons (with the ions playing a negligible role), and because they are, as it proves, essentially nonlinear, it was decided that a distinctive acronym was required to emphasize this. The acronym EAW has been previously used to denote waves in the linear wave frequency gap between the electron and ion plasma frequencies, with phase velocities not far from the electron thermal velocity, in contexts ranging from ionosphere observations, possibly in plasmas with appropriate amounts of clearly defined populations of cold and hot electrons, ${ }^{4,5}$ or in plasmas with Landau damping set equal to zero (perhaps because of some special adjustment to the electron distribution function at some designated phase velocity), ${ }^{3,6,7}$ or as a postulated initial plasma distribution function. ${ }^{6,7,9-16}$ Rather than postulating such distributions, when we drove up the distribution function from the initial Maxwellian by using a limited-time PF driver with a chosen pair of frequency and wavenumbers, we found (as described below) that the trapped electrons obtained kinetically were not simply a means of negating Landau damping but an essential oscillation component quite distinct from and equal in effect to the rest of the (untrapped) electrons. It also appeared that it would be quite difficult to produce such waves at very low amplitudes, so they should not be confused with the result of any linear perturbation. The name for this phenomenon should properly reflect this essentially nonlinear kinetic behavior. It was decided (at the suggestion of Afeyan) to call them KEEN waves, standing for kinetic electrostatic electron nonlinear waves. [In a much briefer study at INRSEMT, to be reported elsewhere, similar aspects were examined with a single species of mobile ions for ionic versions of this kind of excitation at frequencies well below the ion plasma frequency. These were (at the suggestion of Afeyan) termed KEIN waves for kinetic electrostatic ion nonlinear waves.]

The rest of this paper is organized in the following way. It begins with a brief review of previous related work (Sec. II) and of the computer model that was used (Sec. III). Next the basic features of KEEN waves are described in two subsections in Sec. IV [basics (A) (Figs. 1-5), formation dynamics (B) (Figs. 6 and 7)]. This is followed by Sec. V on the basic kinetic nature of the KEEN waves, including a comparison with the initial-value results (Figs. 8 and 9). In Sec. VI there is a discussion of a phenomenon of what is here termed "emergent resonance" (Figs. 10-13). The relationship of the results presented here to the original purpose of this work is then reviewed in Sec. VII, with a final summary and some closing remarks in Sec. VIII. (The details of the calculation of trapping time under ponderomotive drive are given in Appendix A, while the details of a particular formulation for trapped particles are given in Appendix B.)

\section{RELATED PREVIOUS WORK}

There are three main research themes bearing on the KEEN waves discussed here. The first theme is that of the EAWs,${ }^{9,10}$ which arose in ionosphere investigations, their existence being inferred from probe data in the ionosphere. (They are usually seen as requiring two-temperature electron velocity distributions with considerable difference between the two electron temperatures to allow their existence with weak damping.) The second theme is that of KEEN waves as realizations of a particular class of the general BernsteinGreene-Kruskal (BGK) equilibria ${ }^{11}$ in 1D uniform plasma, in which there might be a wave train with trapped electrons such that there is no further evolution in some particular frame (the "wave frame") in which the electron velocity distribution function is stationary. In addition to the work already cited, ${ }^{6,7}$ one should also consult Schamel's more recent work $^{12}$ (and references cited there). Some mathematical refinements have also been added by Dorning and co-workers. ${ }^{13-16}$ Lastly there are miscellaneous explorations of the behavior of 1D phase-space vortices ("holes") and the like. ${ }^{17-23}$ For historical completeness one should also note a particular laboratory experiment done in a Q-machine resulting in the creation of a single KEEN-like cell rather than in a long wave train. ${ }^{24}$

There is also a recent publication by Valentini et al. ${ }^{25}$ which bears directly on the kind of work presented in this publication. (While significant aspects of the relation of that work to ours will be briefly discussed here in Sec. VI, a full discussion must be deferred for now.)

The work which is reported here was begun by Johnston at the instigation of Afeyan, and many abstracts on KEEN waves bearing the names of these two individuals have appeared in the Bulletin of the American Physical Society from 2001 to $2006{ }^{26}$ There is one refereed publication ${ }^{27}$ in 2004. Also, an Invited Paper at the Division of Plasma Physics meeting of 2004 was presented on a KEEN wave experiment (and on other topics), ${ }^{28}$ but the full paper was not published. For reasons which need not be discussed here, it was decided to publish the work performed at INRS-EMT separately from the rest, so this paper presents a summary of most of the KEEN wave research which was done at INRS-EMT from 2001 to 2006.

\section{COMPUTER MODEL}

The simplest model containing the basic physics is that of an electrostatic 1D initially uniform Vlasov plasma in a periodic box, with the option of an externally imposed programmable ponderomotive drive. (This externally imposed $\mathrm{PF}$ approximates that which could be produced experimentally by two high-frequency counterpropagating electromagnetic waves.) The parameters of the PF are chosen at will, these parameters being the driving frequency and wavevector, maximum amplitude, and envelope shape as a function of time (including duration, in particular). The temporal envelope of the PF drive consists of two smooth transitions from zero to maximum drive level and back to zero, each with a duration $T_{\text {trans }}$ and a plateau of duration $T_{\text {plateau }}$ in between. While the PF does not directly involve the Poisson equation, its effects on the electron density will necessarily give rise to an electrostatic response mediated by the Poisson equation. In this work the effect of ion motion is generally neglected, although it can easily be added. (As remarked above, ion motion is a necessary part of the KEIN wave phenomena mentioned above, and these are to be reported 
elsewhere.) The normalization of units is the conventional choice for an electrostatic Vlasov electron plasma with an initial Maxwellian velocity distribution of $\left[f \sim \exp \left(-v^{2} /\right.\right.$ $\left.2 v_{\mathrm{Te}}^{2}\right)$ ]. [The thermal velocity is as usual related to the electron temperature via $v_{\mathrm{Te}}=\left(\kappa_{B} T / m\right)^{1 / 2}$.] The natural computational units are thus $V=v / v_{\mathrm{Te}}$ for velocity, $T=\omega_{p} t$ for time [with plasma frequency $\left.\omega_{p}=\left(n e^{2} / \varepsilon_{0} m\right)^{1 / 2}\right], X=x / \lambda_{D}$ for space [with respect to the Debye length $\lambda_{D}\left(=v_{\mathrm{Te}} / \omega_{p}\right)$ ], and $e E /\left(m \omega_{p} v_{\mathrm{Te}}\right)$ and $F_{p} /\left(m \omega_{p} v_{\mathrm{Te}}\right)$ for electric and PFs, respectively (here $E$ is the electric field, $F_{p}$ is the $\mathrm{PF}$ ).

In order to be able to resolve fine details of the electron distribution function evolution we use a simulation periodic box which is precisely only two driver wavelengths long (so that a full wavelength is always seen in the simulation region). This of course means that the spatial aspects of possible behavior equivalent to the spatially modulational instability of electron plasma (Langmuir) waves are suppressed in such short simulations (because relatively close wavenumbers associated with spatial modulation require relatively long simulation domains), although temporal modulation is allowed.

With the aim of obtaining the finest detail of the velocity distribution function, rather than using the commonly used particle-in-cell (PIC) model for the Vlasov plasma with its inevitable noise, we used the simplest (1D, electrostatic, periodic, with nonrelativistic electrons and stationary ions) Eulerian Vlasov fluid (EVF) model as developed initially by Cheng and Knorr ${ }^{29}$ and as further developed by Bertrand and Ghizzo et al. ${ }^{30}$ For those not familiar with EVF codes for 1D plasmas, we note that the computational burdens for the PIC and Vlasov fluid codes are comparable and fine structures in the $(x, v)$ phase space are easily resolved by the latter, without the complications of discrete particle "noise", provided the velocity resolution is sufficiently good. In the $1 \mathrm{D} \mathrm{EVF}$ model, although noise and precision are not severe limits, the velocity resolution requirement near the phase velocity of the wave for very small traveling-wave perturbations can impose a severe computation burden. This large burden for small perturbations is chiefly due to the fact that the fine velocity resolution, although really required only near the wave phase velocity, is actually imposed over all velocity space, since velocity mesh with current algorithms is required to be uniform over velocity space. Also for weak drives much longer simulation times are required. Hence, although initial signals can be very small in principle, one prefers not to make them much smaller than necessary to access the desired physics. (In passing, while EVF can also be done in principle for two velocity dimensions, the computational burden relative to PIC codes increases dramatically because much effort is expended in calculating the velocity distribution density function in regions of velocity space where the density is very low.)

Because we wished to be able to take spatial Fourier transforms easily, we chose to have 512 space mesh points in our space box which was chosen to be exactly two KEEN wavelengths long. (For a typical KEEN wave value of 0.26 for $k \lambda_{D}$, this meant that our space mesh step was thus typically $0.0944 \lambda_{D}$.) The time step was usually chosen to be 0.04 in plasma radian frequency units, so for a typical plasma- normalized KEEN frequency value of 0.37 , the time step was roughly $1 / 425$ of this KEEN period, allowing excellent representation of the highest harmonics of interest. Finally, in Vlasov fluid codes the velocity mesh is also specified, and we chose 1025 velocity mesh points over the range from $-6 v_{\mathrm{Te}}$ to $+6 v_{\mathrm{Te}}$, where $v_{\mathrm{Te}}$ is the electron thermal velocity of the initial Maxwellian plasma, for a velocity interval $\Delta v$ of $(6 / 512) v_{\mathrm{Te}}=0.01172 v_{\mathrm{Te}}$.

Because of the low-noise properties of the EVF model some useful diagnostics are readily available which are not suitable for most PIC code systems. For a particular signal, if the frequency spectrum (or a selected portion of it) proves to be dominated by a single slowly varying frequency component, one can obtain at any time the amplitude of the signal envelope and the instantaneous signal phase (and hence the instantaneous frequency) by using the time quadrature signal. If only a real time signal (say, from a probe) is available, one can readily generate the required time quadrature component using the Hilbert transform [usually via fast Fourier transform (FFT) in time]. ${ }^{31}$ The signal envelope and signal phase are obtained, respectively, as the square root of the sum of the squares of the real signal and the quadrature signal and as the arctangent of the ratio of the real and quadrature signals (provided one arranges for phase continuity as the branch cuts of the arctangent function are encountered). Often one can readily obtain equivalent information from various spatial Fourier transform components with real and imaginary components for which the phase behavior is usually ignored. In this work when we show a frequency it is the frequency of the fundamental spatial Fourier component of the electron charge density, which is available in complex form (real and imaginary components) at each time step and which could be imagined to be available in a scattering diagnostic in an experiment. This frequency is obtained via $\omega_{n}=\left(\phi_{n+1}-\phi_{n-1}\right) /\left(2 \Delta t_{\text {step }}\right)$, where $\phi_{n}$ is the phase at the $n$th time step of the fundamental spatial Fourier component of the electron charge density.

\section{BASIC KEEN WAVE FEATURES}

\section{A. KEEN formation dynamics}

For linear waves which are not unstable (and which thus do not tend to grow without limit), the canonical behavior for drive times which are long compared to the decay time (wave decay for the $\omega$ and $k$ values of interest here is very short) is that, when the drive is turned off, the level of the driven waves is proportional to the drive strength, and changing the drive duration does not affect that. However, for the waves of interest here the behavior is quite different. It turns out that, if the PF drive is of sufficient level and is applied long enough, it produces a KEEN wave with distinctively nonsinusoidal waveform, and this wave then persists for very long time after the drive has been turned off, far longer than the linear decay time, as if the linear (Landau) damping has been destroyed. This striking longevity of the KEEN waves is clearly remarkable and has led to a study of the drive threshold above which the persistent KEEN waves are produced. We found empirically that, for any particular time program (i.e., particular values of $T_{\text {trans }}$ and $T_{\text {plateau }}$ ) we 
used for the PF drive, there is a rough threshold for the PF drive strength, above which KEEN wave can be produced readily and below which no enduring KEEN wave would result. Conversely, for a given PF drive strength, a sufficiently long PF drive envelope effective duration $T_{\text {eff }}$ is required for producing a self-sustaining KEEN wave (here we define the effective drive duration as $T_{\text {eff }}=T_{\text {plateau }}+T_{\text {trans }}$, which is the effective time during which the drive is at its maximum).

The available simulation results suggest that a criterion of necessary drive strength and duration for producing a surviving KEEN wave can be established in terms of the characteristic electron bounce (or trapping) time $\tau_{B}$ relevant to the formation of an electron trapping vortex in $(X, V)$ phase space. When electron trapping is discussed, usually only electrostatic electric fields are involved. In calculating the electron bounce (trapping) time $\tau_{B}$ when both ponderomotive drive and the induced electrostatic field coexist, as here, one needs to obtain the result including the dynamics of coexistence in a self-consistent way. A reasonable concept is to estimate the response of the untrapped particles using linear theory. It turns out that the electron plasma response for untrapped electrons proves to be well approximated by linear theory over a sufficiently long (up to $\tau_{B} / 2$ ) time during the drive. The resulting normalized electron bounce (or trapping) time $\tau_{B} \omega_{p}$ in the plasma is obtained (see Appendix A) as $\tau_{B} \omega_{p}=2 \pi \omega_{p} / \omega_{B}=2 \pi\left[\left(1+\chi_{e}\right) / k \lambda_{D} a\right]^{1 / 2}$, where $a$ is the normalized (dimensionless) ponderomotive drive amplitude and $\chi_{e}\left(\omega / \omega_{p}, k \lambda_{D}\right)$ is the electron susceptibility. The factor $1+\chi_{e}$ is a large correction at the low frequencies for the case at hand. The plasma response is essentially that of an imperfect but fairly effective shield. For our usual parameters $\omega / \omega_{p}=0.37, k \lambda_{D}=0.26$ we have $\chi_{e}=-1.21+9.59 i$, and the normalized trapping time is then $\tau_{B} \omega_{p}$ $=2 \pi[9.6 /(0.26 a)]^{1 / 2}=38.2 a^{-1 / 2}$. This is the formula used to indicate $\tau_{B}$ in Figs. 1-3 (and later for Figs. 6 and 7).

First, in Fig. 1, one has a typical subthreshold result when the drive is evidently unsuccessful in producing a longlived KEEN wave. The trapping period $\tau_{B}$ [as indicated by the length of the double-headed arrow labeled $\tau_{B}$ in Fig. 1, between the EF (electrostatic force) and PF (ponderomotive force) frames] for the net force during the drive is in this case considerably greater than the effective drive duration (about twice) and the drive is ineffective. For unsuccessful drive cases like this, after the drive is switched off, the electrostatic field in the plasma becomes far less than the value induced during the drive, being rather comparable to the net force during the drive, and decreases in an irregular manner. Note also that during the drive period, as predicted from the linear response theory, the net force acting on electrons $(\mathrm{EF}+\mathrm{PF})$ is far less than either the drive or the resultant electrostatic force EF, which in fact acts mostly to cancel the ponderomotive drive. To produce a KEEN wave one would have to drive harder or longer or both.

The probe results for the case when the drive is sufficiently strong to produce a sustained KEEN wave are shown in Fig. 2, obtained with a drive which is five times stronger than that of Fig. 1. For this drive the trapping time is found to be about the effective duration of the drive. For successful

\section{Subthreshold Drive $a=0.01$}

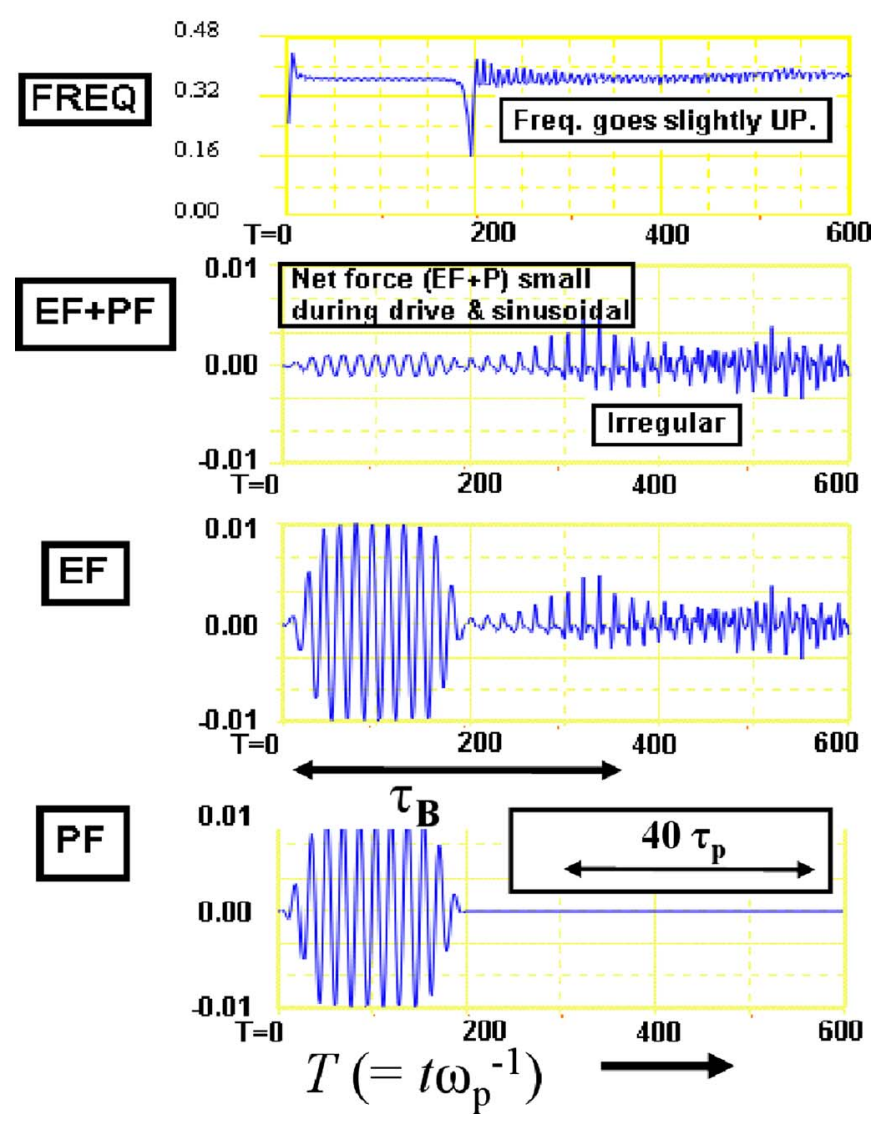

FIG. 1. (Color online) Unsuccessful KEEN wave field results for subthreshold drive (plateau drive time of $150 \omega_{p}^{-1}$ (transitions of $50 \omega_{p}^{-1}$ ), $t_{\mathrm{FP}}=150 \omega_{p}^{-1}$ ), with the trapping time $\tau_{B} \approx 380 \omega_{p}^{-1}$ (as shown by double-headed arrow below the EF plot) being about 2.5 times the effective drive duration. Shown (vs $T=t \omega_{p}^{-1}$ ), from the top down, are the frequency (FREQ), the net force $(\mathrm{EF}$ and $\mathrm{PF}$ or $\mathrm{EF}+\mathrm{PF})$, the $\mathrm{EF}$, and the ponderomotive drive force $(\mathrm{PF})$.

drive cases like this, the net force $(\mathrm{EF}+\mathrm{PF})$ initially follows the linear theory prediction, being far less than the drive PF or the electrostatic force EF, but, at about half the trapping period $\tau_{B}$, when the nonlinearity becomes significant, the net force on the electrons begins to rise dramatically, approaching the sustaining amplitude and shape before the drive ends.

We found that the electron trapping and the associated nonlinearity, essential for the formation of a self-sustaining KEEN wave, is fairly well established during the driving phase by $t=\tau_{B} / 2$, but that to reach a well-established KEEN state, one should at least continue to drive the plasma to about $t=\tau_{B}$. This is the threshold criterion we adopt for the PF-driven KEEN waves. As shown directly in Fig. 2 (and also in Fig. 3 to be discussed next), between $\tau_{B} / 2$ and $\tau_{B}$ the waveforms of the plasma field become markedly nonsinusoidal, indicating nonlinearity, and there is also (in Figs. 2 and 3) a very characteristic change in the net force which becomes comparable to the maximum ponderomotive driving force. Further increasing the drive amplitude nearly always gives little increase in KEEN amplitude. (We return to this topic in Sec. VI, when discussing emergent resonance.)

In Fig. 3 the behavior during and just after the driving phase is examined in greater detail. In Fig. 3(a) the energy 


\section{Adequate Drive $a=0.05$}

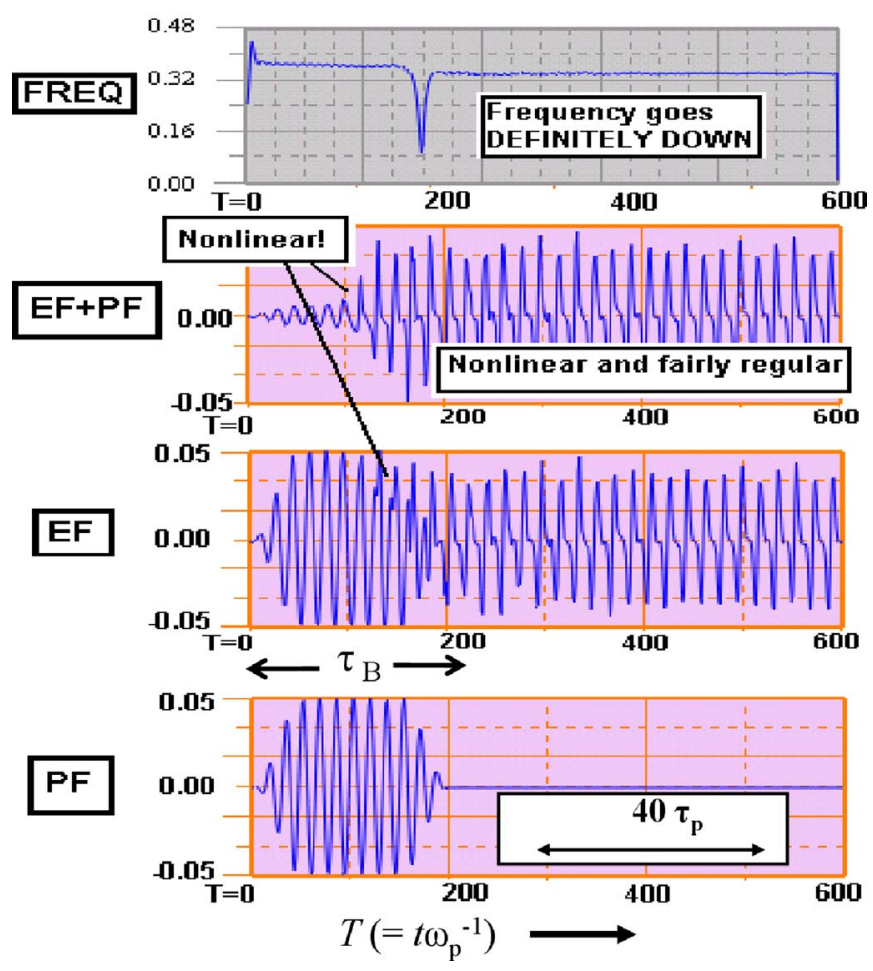

FIG. 2. (Color online) Successful KEEN results similar to Fig. 3 except the drive is now adequate $(a=0.05)$, with the trapping time $\tau_{B}$ (shown by the double-headed arrow) being about equal to the drive duration. Now the result of the drive is to produce a long-lived KEEN wave. As often happens with KEEN wave drive, the final frequency is slightly reduced from the drive frequency.

absorbed in the system is shown to grow linearly with time during the first part of the drive up to $\tau_{B} / 2$, at a rate which is given by the linear susceptibility calculation [power in $\left.=\left|\mathrm{PF} /\left(1+\chi_{e}\right)\right|^{2} \operatorname{Im}\left(\chi_{e}\right) / 2 \omega\right]$. After that, i.e., between $\tau_{B} / 2$ and $\tau_{B}$, the absorbed energy grows noticeably faster than the linear rate, presumably due to additional energy delivery to trapped particles. During this time the high spatial harmonics of the plasma charge density become very noticeable, as shown in Fig. 3(b), as the KEEN's net force nonsinusoidal waveform is set up, as seen in Fig. 3(c). This change seems essential to the transition to a free-running KEEN wave. If one examines Fig. 3(c) with care, the last rather rapid change in the net force waveform is associated with a phase displacement of the electric field which begins to be delayed and to approach the ponderomotive peak. This produces much larger but more highly localized peaks in total field, leading to the formation of the highly nonsinusoidal KEEN structures.

\section{B. Basic KEEN waveforms}

Although the KEEN waves we discuss are driven up using a spatially purely sinusoidal traveling-wave PF driver, it was noticed from the outset that when we have obtained a self-sustaining KEEN wave (by which is meant one that survived long after the drive had ended) the waveform of the KEEN wave thus produced always turned out to be very
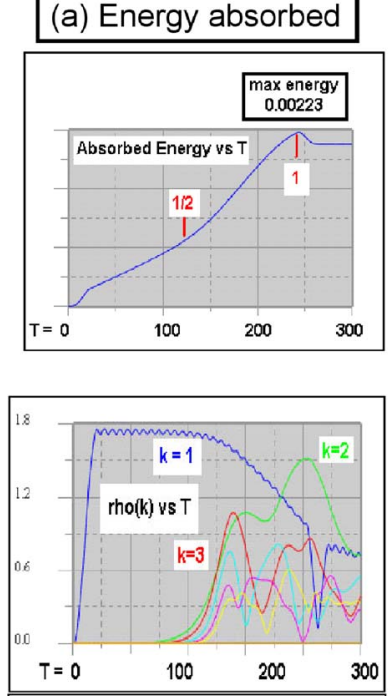
(c) Net Force increases at $1 / 2$ trapping time

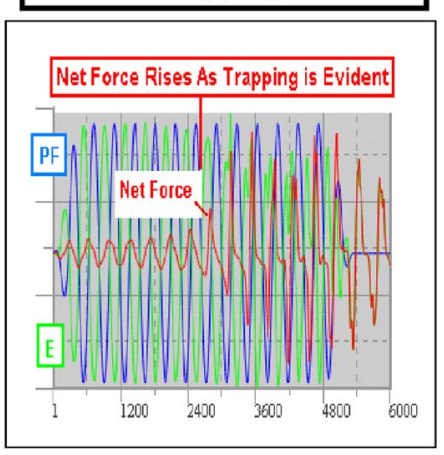

(b) Charge density harmonics vs $T$.

FIG. 3. (Color online) (a) Energy grows at a linear rate $\left[\sim 1 /\left(1+\chi_{e}\right)^{2}\right]$ until trapping becomes significant at half trap (bounce) period $\tau_{B}$, i.e., $\tau_{B} / 2$ (marked as $\frac{1}{2}$ at $T=121$ ), and grows more quickly afterwards. [Pump stops at $242\left(=\tau_{B}\right.$, marked 1).] (b) Harmonics start after $\frac{1}{2}$ trap time. (c) After about $\frac{1}{2} \tau_{B}$, the net force rises and becomes nonsinusoidal and electric field becomes slightly smaller and much more nonlinear.

nonsinusoidal, as shown in Fig. 4. This nonsinusoidal waveform is a clear indication that essentially nonlinear physics is involved (hence the emphasis on nonlinear in the acronym KEEN). Strong nonlinearity is not surprising since, as we will see below, particle trapping is absolutely central to KEEN formation. [This behavior is in contrast to the behavior of the usual Langmuir plasma wave of modest wavenum-

\section{Typical KEEN E-field Non-Sinusoidal Waveform}

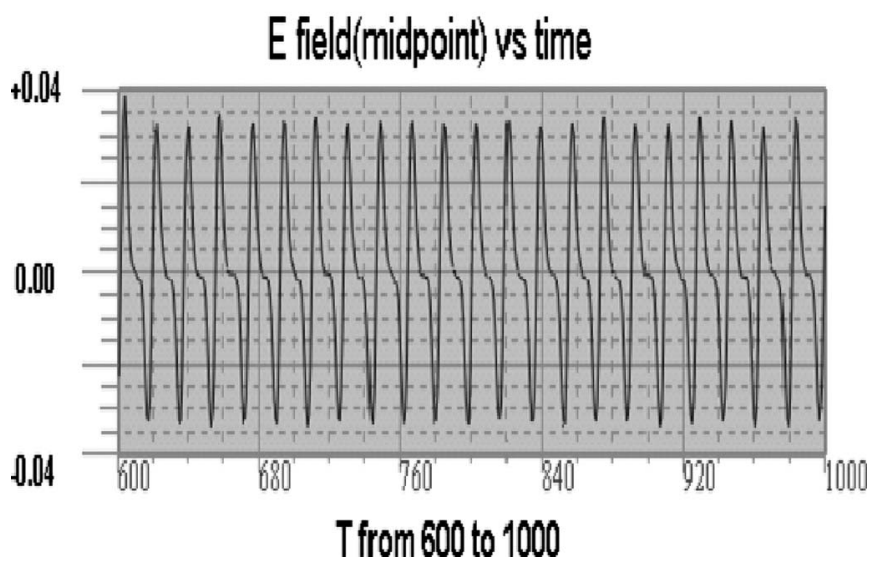

FIG. 4. The sharp rises in E-field are due to the deep narrow hole in the middle of the phase-space vortex passing over the central probe. The waveform is very nonsinusoidal but apparently remarkably stable with little change from one cycle to the next. 


\section{Stable Non-sinusoidal KEEN $f(X, V) \lambda$ $=3.84 \lambda_{\mathrm{D}}(=256 \Delta \mathrm{x}$ cells $)$}

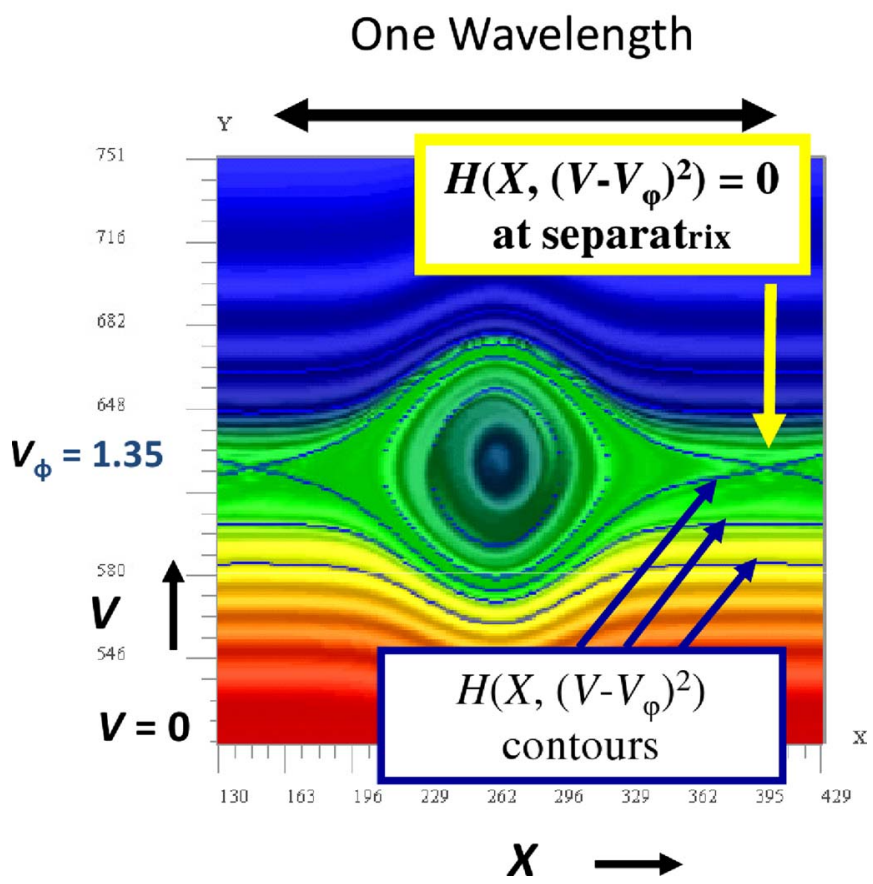

FIG. 5. (Color online) Typical phase-space $(X, V, T)$ example of a stable nonsinusoidal KEEN wave distribution function snapshot (in banded rainbow presentation) late in time, $f(X, V, T=1000)$. Note that the density contours are indeed parallel to the contours of constant wave-frame total energy, as expected from the BGK concept. The PF drive dispersion parameters are $W\left(\omega / \omega_{p}\right)=0.37, K=k \lambda_{D}=0.26$, phase velocity $V_{\varphi}=\omega / k v_{\mathrm{Te}}=0.37 / 0.26$ $=1.42$. The $\mathrm{PF}$ drive envelope parameters are (plateau) $=\langle\mathrm{PF}\rangle / m v_{\mathrm{Te}} \omega_{p}$ $=0.052$, with a pulse shape $\left(\omega_{p} t\right)$ of $0-50$ half-sine up, 50-150 flat, 150-200 half-sine down. After the drive ends the frequency goes from 0.37 to 0.35 , so $V_{\varphi}$ drops to 1.35 .

ber $\left(k^{2} \lambda_{D}^{2} \ll 1\right)$, which can exist at very low amplitudes with little trapping and with quite sinusoidal waveforms even at significant strength.]

The effects of the nonsinusoidal waveform are seen to be significant if one looks at the electron velocity distribution function in its $(X, V)$ phase space over a wavelength for a typical KEEN wave, as shown in Fig. 5. The normalized wave parameters for the drive used for Fig. 5 are as follows: $W \equiv\left(\omega / \omega_{p}\right)=0.37, \quad K \equiv k \lambda_{D}=0.26, \quad V_{\text {phase }}=\omega / k v_{\mathrm{Te}}$ $=0.37 / 0.26=1.42$. The PF drive plateau value was $a=0.052$, the $T_{\text {rans }}=50$, and $T_{\text {plateau }}=150$, for a total duration of 250 .

In the phase-space snapshot of Fig. 5, the banded rainbow presentation shows the contours of constant density, and how they lie parallel to the contours of constant total energy $H$ (where $H=\frac{1}{2}\left(V-V_{\phi}\right)^{2}+\Psi$, as expected from the BGK concept. (In Fig. 5 the $H=0$ separatrix value is shown as a green line as well as two for $H>0$ and two for $H<0$.) It is useful to note that, to the extent that the KEEN waves resemble perfectly stationary BGK waves in the wave frame, the constant density contours (and, in particular, the ones that go through the $X$ points) reveal the form of the square root of the electrostatic potential. In Fig. 5 the form of this contour is clearly far from the half-wavelength sinusoid which one would obtain from the square root of a sinusoidal potential.
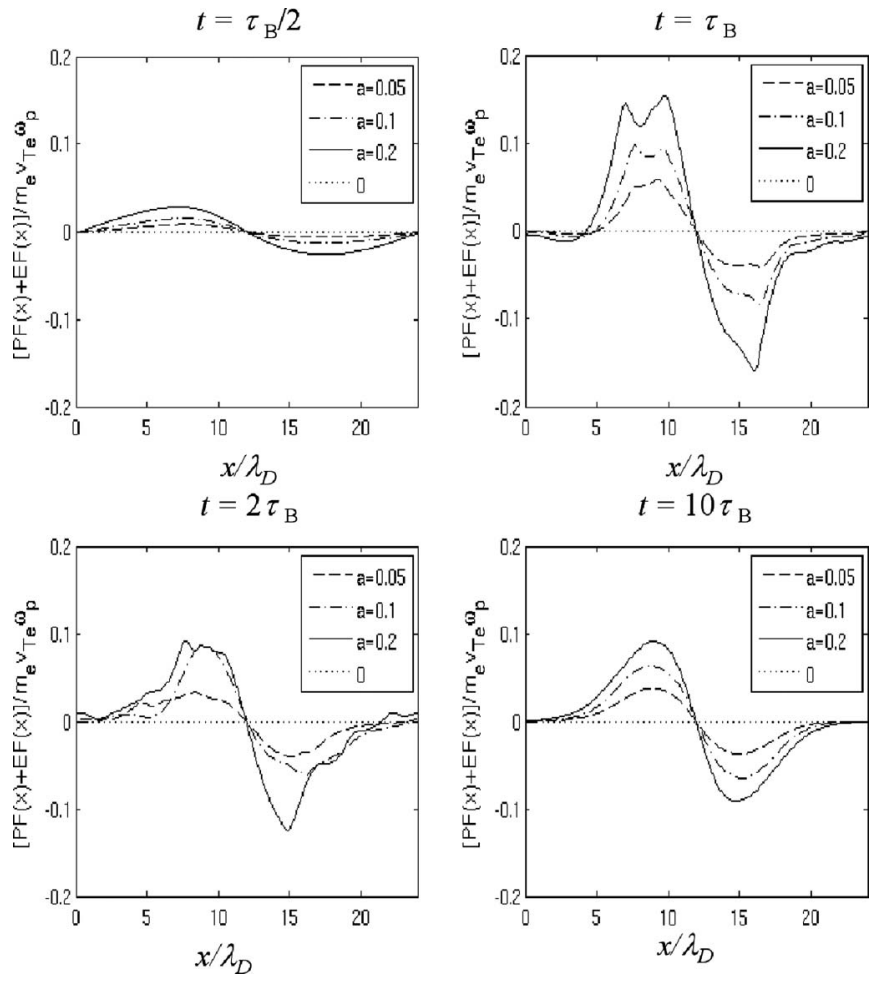

FIG. 6. Evolution of successful $\mathrm{KEEN}$ force wave shapes $[\mathrm{EF}(X, T)$ $+\mathrm{PF}(X, T)]$ during and after the drive. Snapshots (at $\left.t / \tau_{B}=1 / 2,1,2,10\right)$ are for drive levels (FP) of $a=0.05,0.1,0.2$ and corresponding normalized trapping times of $\omega_{p} \tau_{B}=170,120,85$. The general wave shapes at times scaled to the trapping time $\tau_{B}$ resemble each other quite closely. The amplitudes (and particularly the ultimate KEEN wave amplitude) scale monotonically with the drive amplitudes (but less than proportionately).

\section{KEEN approach to asymptotic form and $\tau_{B}$ scaling}

After determining that the key parameter in KEEN formation is the trapping time $\tau_{B}$, it is a natural question as to whether this time is the useful way to scale the evolution of KEEN waves. In order to test this idea we chose three characteristic drive strength values $(a=0.05,0.1$, and 0.2$)$ over a considerable range in strength, applied in each of these drives for a time equal to their corresponding value of $\tau_{B}$ $\left(\omega_{p} \tau_{B}=170,120\right.$, and 85 , respectively $)$ and plotted the results for $t / \tau_{B}=\frac{1}{2}, 1,2,10$. The results are shown in Fig. 6, and they seem to confirm the $\tau_{B}$ scaling.

The general shape of the characteristic nonsinusoidal total-force waveform at late times (the long-time set of images in Fig. 6) is generally similar for all the three KEEN waves produced. That force shape corresponds to relatively narrow potential wells with wide shoulders. One might well have expected naively that the characteristic ultimate waveform would be sinusoidal at weak drives and more nonlinear at strong drives. However, we see from Fig. 6 that the ultimate waveforms of the successfully generated KEEN waves have strikingly similar, markedly nonsinusoidal shapes in their ultimate states ( amplitudes.

The evolution of the electron velocity distribution function is shown in Fig. 7, with the phase-space snapshots taken at the same times $t / \tau_{B}=\frac{1}{2}, 1,2,10$ as the waveform images 

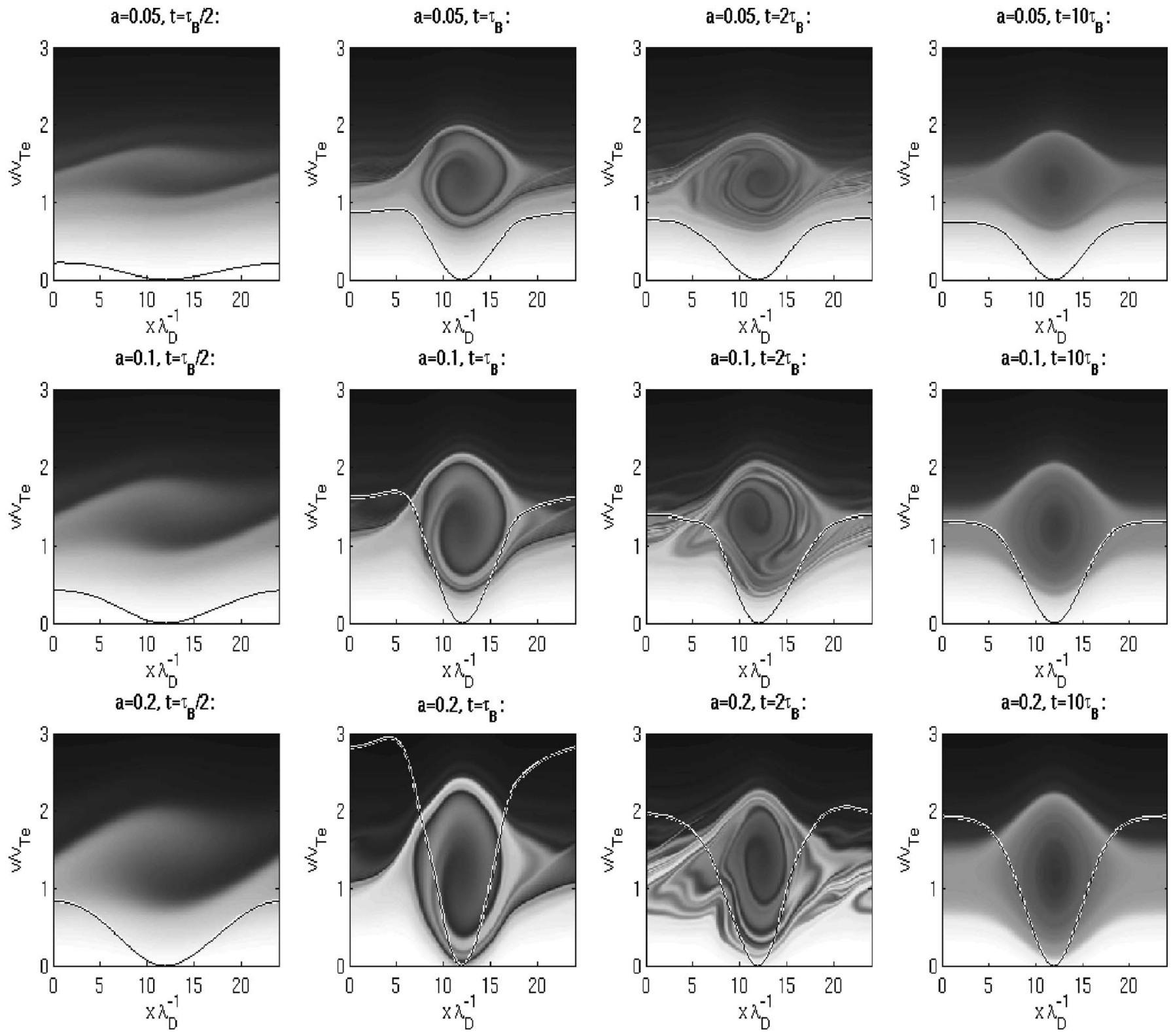

FIG. 7. Phase-space $(X, V)$ images of electron velocity distribution function during the drive-up period $\left(t \leq \tau_{B}\right)$ and after at the same times as for Fig. 6 (namely, at $t / \tau_{B}=1 / 2,1,2,10$ ). The total-force potential profiles for each time frame are superposed for convenience.

of Fig. 6, with their total-force potentials superposed. As was found for the waveforms, it is seen that the distribution functions resemble others with different drive strengths at the same value for $t / \tau_{B}$ rather than those at the same drive strength and significantly different values for $t / \tau_{B}$. For the evolution of the distribution function the $t / \tau_{B}$ scaling seems to work quite well, so one can describe the evolution of a KEEN wave fairly generally as a function of $t / \tau_{B}$.

The development of the distribution function up to $t / \tau_{B}=\frac{1}{2}$ reflects a very normal early evolution of the distribution function. Between $t / \tau_{B}=\frac{1}{2}$ and $t / \tau_{B}=1$ one sees the development of a well-organized vortex in phase space which would seem likely to survive, just as the drive is being terminated. With the drive already turned off for some time (about $\tau_{B}$ ) at $t / \tau_{B}=2$, the phase-space vortex is showing some complicated evolution with fine ripples and structures and related structure in waveforms, as shown in Fig. 6. By $t / \tau_{B}=10$ the distribution function in Fig. 7 and the waveform in Fig. 6 appear to have evolved to relatively smooth states which resemble BGK-like states, at least superficially. The question of whether there is a continuing evolution to an ideal BGK state or whether fluctuations about this ideal state do not die out with time must be left to investigations with more sophisticated diagnostics than those employed here.

The content of Fig. 6 is that, when scaled to the trapping time the formation of a typical KEEN structure is very much the same up to a single trapping period. The trapping time scales as the inverse square root of the driving force, so that for very weak drive the trapping time is very long. Consistent with this, the KEEN velocity perturbation extent around the drive phase velocity (e.g., the region including trapped electrons) scales as the square root of the driving force, so that for very weak drive, the size of the trapped velocity region is very small. 
The behavior after a trapping period has not yet been examined in detail, but what happens to a driven KEEN wave after the trapping period has been reached can be outlined as follows. Usually, if the drive is extended beyond the electron trapping period $\tau_{B}$, the amplitude of the wave being driven begins to oscillate on the scale of the trapping period, starting with a decrease to a lower value (usually down to $80 \%$ or so) and then back up again to the original maximum value attained at $T_{\text {drive }}=\tau_{B}$. Driving past one $\tau_{B}$ does not increase the maximum amplitude of the resulting KEEN wave. Turning off the drive in a time short compared with the electron trapping period (but after a duration long compared with a trapping period) leaves the KEEN wave amplitude at about its value when turn-off was initiated. What appears to be happening is that a phase mismatch between the PF drive and the plasma response develops over time due to nonlinear effects and energy is subsequently exchanged in oscillatory fashion (with period of the order of $\tau_{B}$ ) between the driver and the KEEN wave without steady accumulation in the latter, and this exchange ceases when the drive is terminated. The ultimate amplitude of the KEEN wave is defined by the phase in this oscillatory energy exchange at which the drive has been turned off. However, although it was not realized when this behavior was being studied by us earlier, in exceptional cases continuing energy accumulation by the KEEN wave can occur and this is discussed in Sec. VI.

\section{KINETIC NATURE OF KEEN WAVES}

The evidence has been presented that a fairly steady state in the wave frame can indeed be obtained by applying a prescribed ponderomotive driver for a limited time. It is time now to turn to some diagnostics which indicate how, so to speak, the KEEN wave maintains its equilibrium. For this aspect, as well as investigating the KEEN waves driven up ponderomotively, it was judged appropriate to include in such analysis some examples of KEEN waves obtained in the same way used in nearly all the previous work. There one seeks to obtain some approximation to an ideal state for a steady wave with trapped particles, where the system appears perfectly still in the frame traveling at the wave speed, in what is normally known as a BGK state. ${ }^{11}$ This common method was simply to begin by setting up by some BGK analytic prescription for the velocity distribution in phase space, as an approximation to the desired final state and see if this state survived. . $^{3,72-18}$

In order to be able to respond to comments related to the constructive or initial-value method, it was examined in some detail, of which only the most essential points are given in this paper. One should recall that the BGK concept, when reduced to its essentials, is the assertion that, for steady state behavior in the wave frame, the distribution function in that frame should be a function of space only implicitly, i.e., through the behavior of any space-varying potential in relevant canonical particle conserved quantities. In the $1 \mathrm{D}$ electrostatic case of interest here this means that the distribution function must be a function only of the particle total energy (from which the velocity may be calculated when the elec- trostatic potential is given). [It is often not appreciated that BGK (Ref. 11) did not assert anything about whether BGK modes actually exist and whether they are stable in a particular velocity frame. The assertion was only that if such stationary solutions exist, then they must follow the BGK rule. One should therefore always test such a BGK candidate for stability with a proper simulation, to see that it will endure close to the original formulation.]

What we have found is that, as shown in Fig. 5, electron distribution density contours in phase space seem to follow quite closely the contours of the total particle energy, as they should in a BGK equilibrium. On closer examination, however, deviations from this ideal are seen which are not stationary in the wave frame and whose ultimate fate is uncertain. Hence in this work, at least, the BGK model is regarded as an extraordinarily useful ideal which is probably very difficult to attain, rather than as an attractive state to which the system evolves.

When choosing an initial distribution function according to the BGK canon, one has considerable freedom to choose the details. Naturally, for a given potential the distribution results of the different functional recipe choices for trapped particles will differ from each other. After considering the explicit recipes given by Holloway and Dorning [Ref. 14, in Eq. (84)] and by Eliasson and Shukla [Ref. 32, in Eq. (9)], they were not used here because those recipes tended to give distribution function velocity wells whose depths were much shallower (for the same well velocity widths) than those seen for PF-driven KEEN waves. The distribution function form that was adopted here was a significant modification of a little-known form given by Allis. ${ }^{33}$ This modification was necessary to avoid unphysical discontinuities in the result (see Appendix B), and this modified form was hence dubbed the Allis-Johnston (AJ) formula. This recipe was developed in a very organic way from a Maxwellian, with which our drive program began, and, most gratifyingly, the velocity distribution function was found to agree quite well with KEEN velocity distributions for the same potential, as shown in Fig. 8 for the deepest part of the potential well in a typical case.

In the top row of Fig. 9 are shown a typical driven KEEN wave (on the right) with an equivalent $\mathrm{AJ}$ result (on the left) for the same maximum potential but using a sinusoidal assumption (almost invariably used in BGK work) for the initial potential waveform. While the section across the maximally perturbed region may look the same (as shown in Fig. 8), the AJ construction begun with a sinusoid usually remains superficially sinusoidal and so the separatrix looks rather like half-wavelength sinusoid. (However, the net charge waveform, which is proportional to the second spatial derivative of the potential, is distinctly nonsinusoidal, resembling a cnoidal waveform rather than a sinusoid.) In contrast to this, the KEEN wave driven up from a sinusoidal driver has here been shown to be much more nonsinusoidal. Thus, while constructing BGK moving equilibria using a suitable recipe may give something resembling a KEEN driven up in specific way, the actual waveforms will not correspond well unless the initial waveform used for initiating a BGK-like state is copied from an actual driven KEEN result.

In the bottom row of Fig. 9 are plotted the spatial varia- 


\section{Distribution function comparisons: A-J vs KEEN}

$X$ - point slice

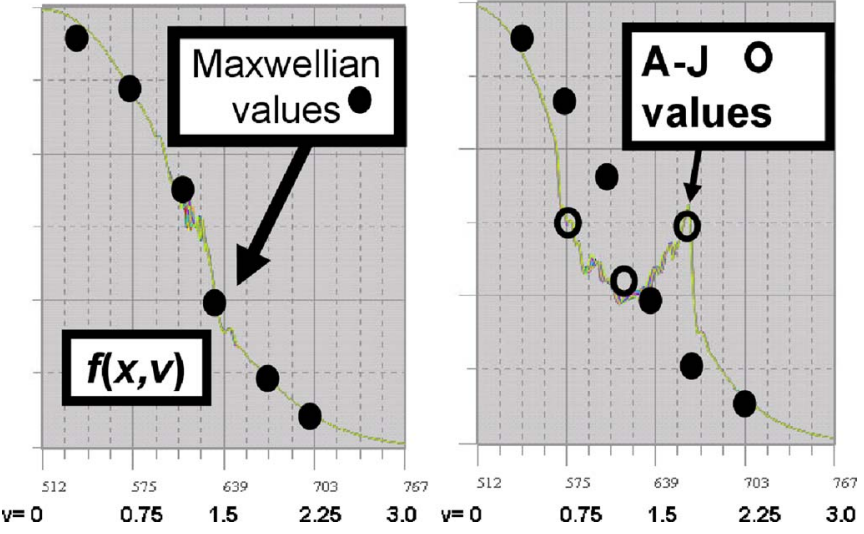

FIG. 8. (Color online) Theorist-created versions of BGK wave train states $f\left(X=x / \lambda_{D}, V=v / v_{\mathrm{Te}}, T=\omega_{p} t\right)$ are clearly artificial, but they can persist with little change in time. An AJ sinusoidal BGK constructed to match a particular KEEN wave at the center fits the Vlasov code distribution (solid line) quite well and is also quite steady, as shown here at a potential $X$ point and at a potential minimum ( $O$ point). Either of the forms can be used to check how the wave is structured, as shown in the next figure. (Normalized parameters used here were typical values: $W=\omega / \omega_{p}=0.35, K=k \lambda_{D}=0.26, a$ $=0.05$. The KEEN maximum trapped velocity width was found to be $D V$ $=0.36$, corresponding to a maximum KEEN $E$ of 0.062 .)

\section{Allis-Johnston}

$\omega / \omega_{\mathrm{p}}=0.4, k \lambda_{D}=0.26$

\section{KEEN, PF Drive}

$$
a=0.02, \omega_{\mathrm{p}} t_{\text {drive }}=200,
$$$$
\text { at } \omega_{\mathrm{p}} t=2000
$$

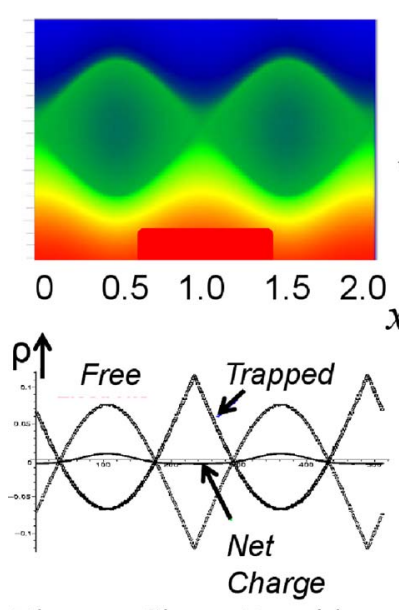

Electron Charge Densities

Free, Trapped, Net

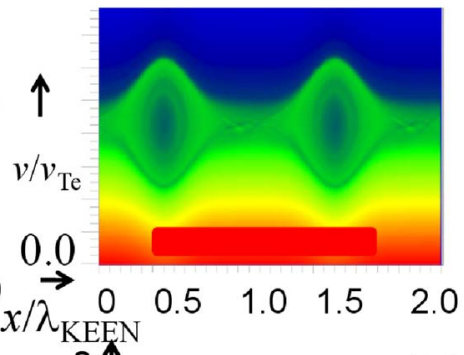

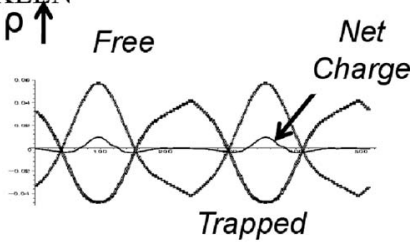

Electron Charge Densities Free, Trapped, Net
FIG. 9. (Color online) On the left, an AJ BGK construction (see text) with sinusoidal waveform was chosen to match a ponderomotively created KEEN wave (on the right) in strength, spatial period, and phase velocity. In both cases, comparing the charge contributions of the free and trapped electrons they are seen to be very nearly equal and opposite (with the trapped electron density dominating slightly at the bottom of the wells), so the magnitude of the net charge variation is very much smaller than that of either the free or the trapped electrons. [Note that the scale is $\lambda_{\text {KEEN }}$ $\left.=(2 \pi / 0.26) \lambda_{D}=24.166 \lambda_{D}.\right]$ tion component of the space charge of the trapped and free electrons and also of the net charge. One can readily see the basic construction of the KEEN wave and its AJ counterpart as a moving phase-space vortex of trapped electrons which is nearly completely neutralized by the plasma shielding response of the free electrons so the net charge is very small. In this aspect the KEEN wave two-electron species dynamics resemble the ion sound wave (IAW) two-species (electrons and ions) dynamics rather more than the usual electron plasma Langmuir wave (where vacuum displacement current is balanced with electron current). In both KEEN and IAW cases (i) the two species react quite differently to the electric field, (ii) the electric field acts mainly to couple the charge variation of two species together, and (iii) the net charge variation is much less than the variation of the charge density for each species. However, unlike ion sound waves that do exist in a fluid description, the KEEN waves are essentially kinetic in that, in order to exist, they require an appropriate population of well-trapped electrons (a kinetic phenomenon that does not occur at all in a fluid description).

Now, for the ion sound wave, the electrons form one dynamic species and all the ions form the other, the motion being thus ambipolar for modest wavenumbers. Linear theory for weakly damped long-wavelength ion sound waves allows one to calculate the relative contributions of the Poisson field, the electron shielding, and the free ions as, respectively, $1:+1 /\left(k^{2} \lambda_{D}^{2}\right):-\sum \omega_{\mathrm{pi}}^{2} / \omega^{2}$, with the first term being relatively small compared with the other two. Similarly, for a strongly two-temperature electron distribution there is also a weakly damped electron sound mode, the EAW, whose small signal behavior can be obtained from perturbation theory and which is comparable to a KEEN wave, with hot electrons partly shielding the cold electron plasma density variation. $4,5,9,10$ From the linear theory for this strongly twotemperature case, the ratios of the vacuum contribution to the fast electron contribution to the cold electron contribution are $1:+1 /\left(k^{2} \lambda_{D \text { hot }}^{2}\right):-\omega_{p \text { cold }}^{2} / \omega^{2}$, with the first term having the much smaller magnitude. Returning to KEEN waves, whether driven up ponderomotively or created via BGK, the two dynamic species are the trapped electrons and the free electrons and they seem to be behaving in a similar way. However, unlike for ion sound waves and EAW, for the KEEN waves in a Maxwellian plasma there is as yet no linear perturbation theory to apply to check the smallamplitude behavior. To sum up, it thus seems that in KEEN waves in Maxwellian plasmas (however formed) the trapped electrons play the role that hot electrons play in twotemperature EAWs, while the free electrons play the role of the cold electrons but with trapping eliminating the severe Landau damping which would otherwise obtain for comparable (or identical) electron temperatures.

The BGK candidates produced with our BGK construction method were tested for stability and stationarity (in their waveframes) by a close examination of their further temporal evolution with a suitable noiseless Vlasov simulation, to which the BGK candidates were given as initial states. It is shown that the constructed BGK candidates remain stable when allowed to evolve; however, the modest continuing small fluctuations seen in the ultimate states of KEEN waves 


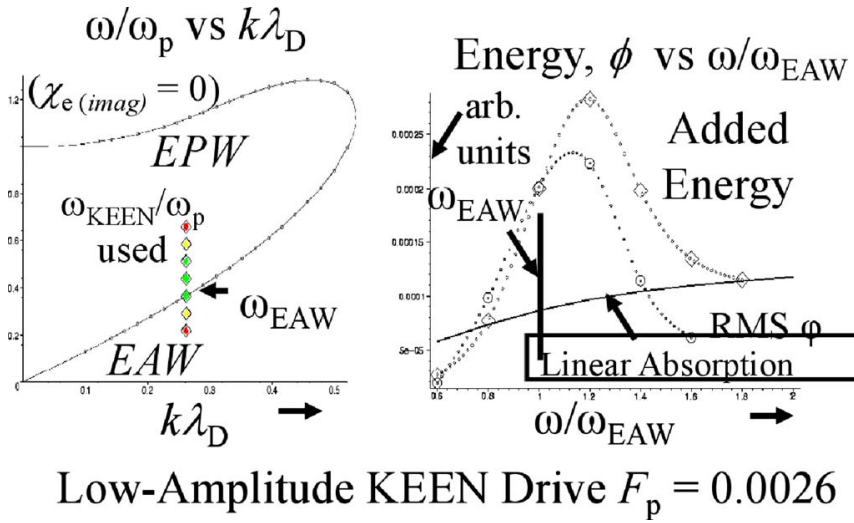

FIG. 10. (Color online) KEEN Amplitudes for $k \lambda_{D}=0.26$, with a common value for the low-amplitude drive $(a=0.0026)$ but with various drive frequency values. These correspond to $\omega / \omega_{p}$ values from 0.2 to 0.7 , corresponding to $\omega / \omega_{\text {EAW }}$ values from 0.6 to 1.8 . Note that, as expected from linear theory, KEEN waves can be produced over a broad range of frequencies for a given wavevector and do not show a narrow resonance around $\omega_{\text {EAW. }}$.

were also observed in all our BGK attempts. Perhaps making an ideal BGK mode which is absolutely stationary in its moving frame is difficult or even impossible.

We note as a side issue an instability that was seen by Valentini et al. (Ref. 25, their Fig. 7) as merging of phasespace vortices. [This was observed in their investigation of what we will term (see below) the emergent resonance phenomenon, which will be discussed later.] The instability of merging of phase-space vortices was discussed long ago by Berk et $a l^{34}$ and later by Bertrand et al. ${ }^{35}$ and very recently indeed in SRS-related work by Albrecht-Marc et al. ${ }^{36}$ In all the cases cited so far where such vortex merging was seen, the size of the vortices before merging occurred was quite large.

In the cases we discuss here the KEEN vortices are usually quite modest in size and vortex merging was not seen. However, this might be due to the lack of a noise source in a Vlasov fluid code or simply to the fact that in our concentration on typical features of KEEN formation and survival we have not run appropriate simulations for the rather long times required for the vortex-merging instability to appear. (In fact some vortex merging had been observed in some very long runs, but the phenomenon was put to one side, without the data being kept, in pursuit of our more immediate objectives.) It may also be the case that the nonsinusoidal KEEN waveform with relatively little field near the separatrices (compared with a sinusoid) slows any vortex-merging tendencies that there may be.

\section{EMERGENT RESONANCE FOR KEEN WAVES}

As is well known, at the low frequencies of interest here, the linear plasma response has no weakly damped eigenmodes, so one might well expect that there is little selectivity in frequency at a given wavenumber for driving up KEEN, and this is more or less true. In Fig. 10 are shown for reference the $\omega, k$ results [comparable to Fig. 2(b) of Ref. 1] obtained by artificially setting the imaginary part of the linear electron susceptibility equal to zero, thus artificially

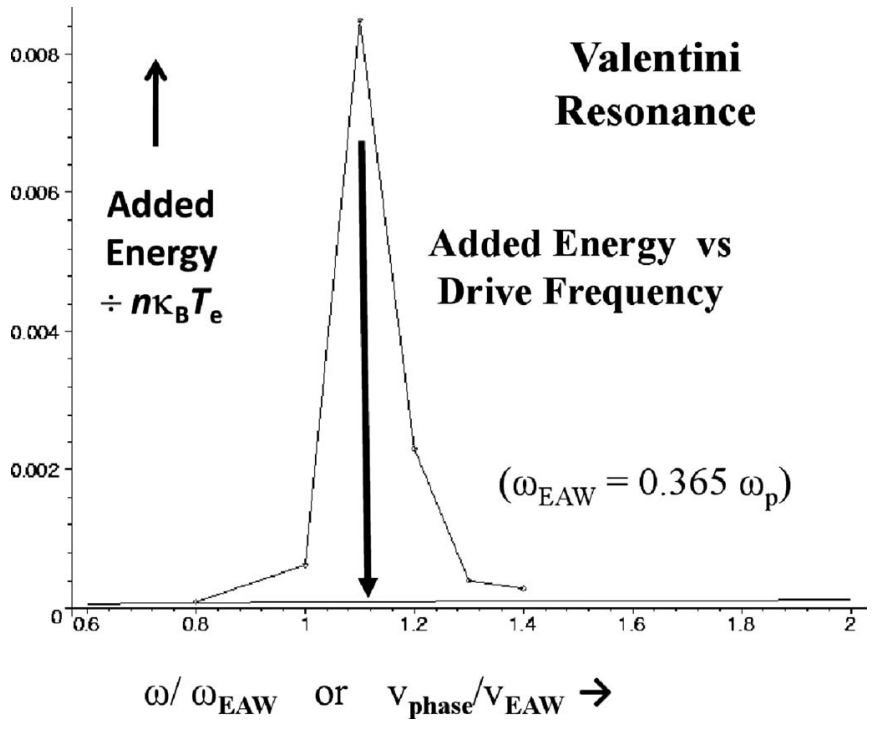

FIG. 11. Although the KEEN wave does not appear resonant under drive for an electron trapping period or so, for low drive maintained for many trapping cycles one can find the emergent resonance of Valentini et al. This is a narrow resonance which emerges at a particular frequency (here at $\omega$ $\left.=1.1 \omega_{\mathrm{EAW}}=0.402 \omega_{p}\right)$ late in time when the result of the same low-level drive at neighboring frequencies has saturated.

eliminating Landau damping. The lower part of this "thumb" curve is what has been called the EAW branch. ${ }^{9,10}$ While the EAW curve does give some guidance as to the lowest frequency that can be easily excited as a KEEN mode, it is clear that KEEN modes for the wavenumber shown can be driven up very far away (in $\omega-k$ space) from that locus. The frequency axis is normalized to the EAW frequency, showing that KEEN waves can be readily produced over a 2:1 range of frequencies (80\% of EAW frequency to $160 \%$ of EAW frequency) for this typical drive wavenumber. There is a broad peak in the response [shown in Fig. 10 (right frame) by $\operatorname{rms} \varphi]$, and this peak is significantly displaced upward from the EAW value (by approximately $20 \%$ of $\omega_{\text {EAW }}$ ).

One of the major points of the work presented here as exemplified in Fig. 10 is that the excitation of KEEN waves is a broadband phenomenon, consistent with the heavily damped nature of the linear plasma response at these KEEN frequencies, well below the plasma frequency. In a result which differs remarkably from this broad-band result such as Fig. 10, Valentini et $a l^{25}$ obtained a result for a particular case with very low-drive amplitude which seemed to be in direct contradiction to the typical result of Fig. 10, in that a response was obtained (in their Fig. 3) which was much narrower than that of Fig. 10. Accordingly that particular situation is revisited here and is confirmed and verified in some more detail in Fig. 11. There indeed seems to be a strong contradiction, in that a relative half-height width of about 0.55 is seen in Fig. 10 and one of only about 0.15 in Fig. 11 (which is our version of Fig. 3 in Ref. 25). However, the two apparently contradictory situations are in fact quite consistent with each other, as we show next.

To resolve the apparent contradiction one must examine the time history of the behavior of the amplitude growth under drive. The early history is shown in Fig. 12. At these 


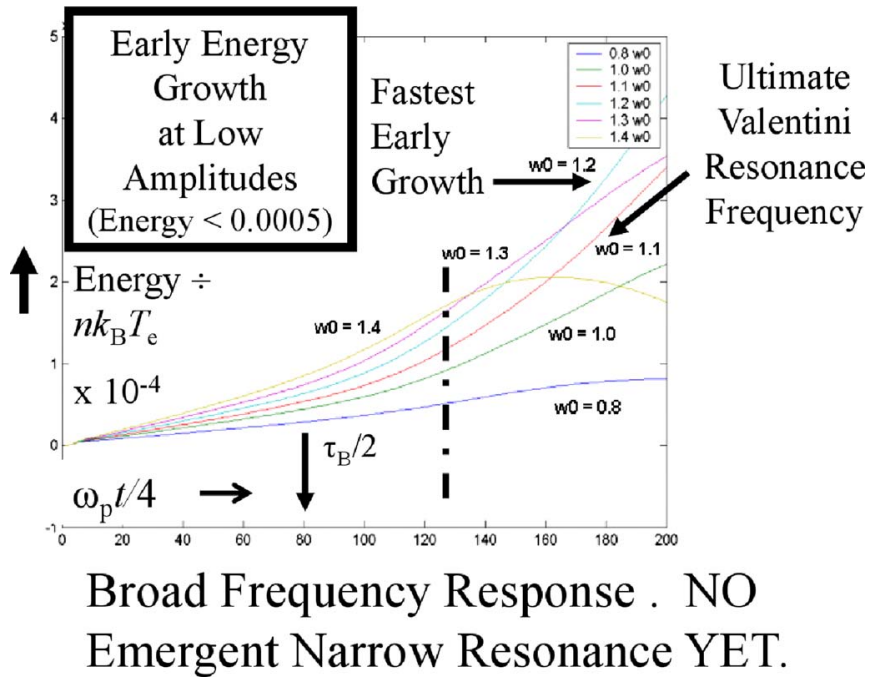

FIG. 12. (Color online) Time history of KEEN wave energy accumulation from the drive for different driving frequencies, ranging from $0.8 \omega_{\mathrm{EAW}}$ to $1.4 \omega_{\mathrm{EAW}}$. Valentini resonance before its emergence (i.e., at early times) shows normal early added energy growth with weak drive $(a=0.0026) . \tau_{B} / 2$ is about 80 on this coarse time step scale of $320 \omega_{p}^{-1}$. (The weak drive continues to $3500 \omega_{p}^{-1}$ or about $5 \tau_{B}$.)

relatively early times (up to about $\omega_{p} t \sim 480$ ) the behavior is seen to be quite broad band in nature, consistent with Fig. 11. However, beyond the trapping period there emerges (in this low-drive case at least) a narrow resonance behavior which is here termed an emergent resonance as shown in Fig. 12 for early times and in Fig. 13 for later times.

It is clear from Fig. 13 that the narrow resonance for (low) drives much longer than the trapping/bounce time $\tau_{B}$ does not appear in the linear phase of the plasma response to the drive but rather emerges after about two trapping periods, in the sense of a frequency-localized (in a narrow frequency range centered in this case at about $\omega_{0} / \omega_{\text {EAW }}=1.1$, where $\omega_{0}$

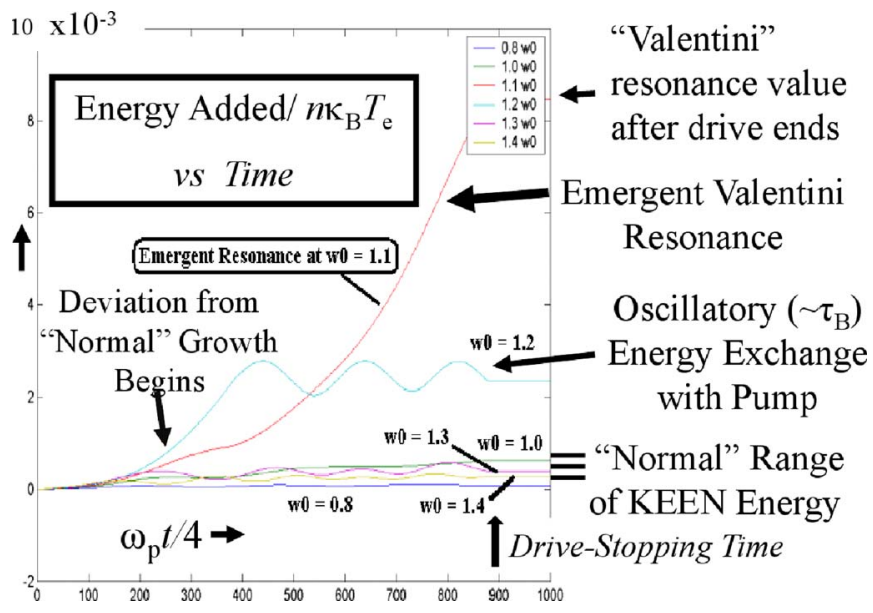

FIG. 13. (Color online) Extended time history of KEEN wave energy accumulation from the drive for different driving frequencies, ranging from $0.8 \omega_{\mathrm{EAW}}$ to $1.4 \omega_{\mathrm{EAW}}$. Emergence of the nonlinear resonance of Valentini et al. is apparent at late times. Long-time behavior of added energy (up to 0.01 for the monotonically growing case) shows how the emergent KEEN resonance observed by Valentini et al. (here at $\omega=1.1 \omega_{\mathrm{EAW}}=0.4015 \omega_{p}$ ) appears at the time where saturation is usually seen at about $2 \tau_{B}\left(\tau_{B} \sim 640 \omega_{p}^{-1}\right.$ or 160 on the scale of this figure). is the driving frequency) immunity to the saturation via trapping oscillation of the kind so clearly seen at neighboring drive frequencies (such as $\omega_{0} / \omega_{\mathrm{EAW}}=1.0,1.3$ ).

Understanding how this comes about will require a much more profound study than has been made so far. It is clear that the effect is related to the basic physics of why the normal saturation of the KEEN wave drive occurs and how this does not occur for the right frequency. What the initial study reported in this section affords is the observation that this saturation is normal but not why it occurs nor why there is a very narrow frequency range where it does not occur. As has been noted above, there is usually a change of KEEN frequency (and phase velocity) as the drive is removed. The emergence of the resonance may well be related to this phenomenon. One may hope that this will become clear in future work.

\section{KEEN WAVE AND THE LABORATORY SCATTERING RESULTS}

At the outset the proximate motivation for this work was the possibility that the KEEN waves that were studied might be an important part of the puzzling scattering signals observed by Montgomery et al. ${ }^{1,2}$ In fact it has indeed emerged that KEEN waves can be driven up fairly easily (in numerical simulations) and the Landau damping overcome (at least in periodic systems). What did not emerge was how the KEEN frequency and wavenumber could be selected by the plasma in the first place. While the narrow-band phenomenon presented by Valentini et al. ${ }^{25}$ might seem to be a candidate, this is an illusion because this emergent resonance behavior only appears after a considerable time of externally driving the plasma at an externally chosen frequency and wavenumber, and so cannot be relevant when asking how the growth process can start in an initially Maxwellian plasma.

In fact it now seems likely that the initial assumption for this work that one begins with a Maxwellian plasma is not a good approximation for the experiment. This is what emerged from the simulation work of Strozzi et al. ${ }^{8}$ where they looked at the plasma as it evolved in the presence of significant SRS, which was in fact the condition in the actual experiment. ${ }^{1,2}$ The reader is referred to the original paper ${ }^{8}$ for the interesting details. Here it suffices to say that their simulation and analysis of that significantly non-Maxwellian plasma (see especially Figs. 10 and 11 of Ref. 8) showed that the "EAW" excitation spectrum was present and was to be expected. The non-Maxwellian nature of the SRS-modified plasma was at the heart of their explanation. Whether significant KEEN waves may occur in other circumstances is a topic which is left for future work.

\section{CONCLUSIONS}

Driving a 1D collisionless Maxwellian (Vlasov) plasma with a sufficiently strong longitudinal ponderomotive driver for a sufficiently long time results in a self-sustaining wave train even for frequencies well below the plasma frequency and at phase velocities somewhat above the electron thermal velocity, which we have termed a KEEN wave as suggested by Afeyan. ${ }^{28}$ In effect the successful drive results in a 
trapped electron distribution well distributed in the trapping well so as not to remove energy from the wave. The criterion linking the drive strength and the drive duration is that the drive duration exceeds the electron bounce time $\tau_{B}\left\{=\omega_{B}^{-1}\right.$ $\left.=\left[\left(1+\chi_{e}\right) / k a\right]^{1 / 2}\right\}$. For a given wavenumber a wide range of KEEN wave frequencies and wavenumbers can indeed be excited. The typical KEEN waveform proves to be very nonlinear and very nonsinusoidal. The basic KEEN structure is kinetic with the trapped and free electron densities almost tracking each other, leaving just enough net charge to support the wave. The nature of the emergent resonance which seems to be an avoidance of saturation remains to be determined. Finally, as to an explanation for the original scattering results $^{1,2}$ which led to this work, it seems likely to be in the work presented by Strozzi et al. ${ }^{8}$

\section{ACKNOWLEDGMENTS}

Tudor W. Johnston acknowledges many discussions with Dr. Bedros Afeyan. Financial support for this work was accorded by the National Science and Engineering Research Council of Canada (NSERC) under a Discovery Grant (General Physics) No. 9613-07.

\section{APPENDIX A: HOW TO CALCULATE THE BOUNCE TIME $\tau_{B}$ WITH PONDEROMOTIVE DRIVE}

The Fourier transform of the linear electron charge response to the combination of ponderomotive potential and plasma electrostatic potential is the following:

$$
\delta \rho_{e}(\omega, k)=-k^{2} \varepsilon_{0} \chi_{e}(\omega, k)\left[\phi_{\mathrm{PF}}(\omega, k)+\phi_{E}(\omega, k)\right],
$$

where $\chi_{e}$ is the electron susceptibility and $\omega$ and $k$ are the frequency and the wavenumber of the response (and the driver), respectively.

The Fourier-transformed Poisson equation with only electron density disturbance $\delta \rho_{e}$ (ions are treated as immobile charge-neutralizing background here) is

$$
k^{2} \phi_{E}(\omega, k)=\varepsilon_{0}^{-1} \delta \rho_{e}(\omega, k) .
$$

Eliminating $\phi_{E}$ in terms of $\delta \rho_{e}$ results in the following equation, from which one gets the relationship between $\delta \rho_{e}$ and $\phi_{\text {pond }}$ and then, upon taking the inverse Fourier transform, that between $\phi_{E}(t, x)$ and $\phi_{\mathrm{PF}}(t, x)$ :

$$
\begin{aligned}
& \delta \rho_{e}(\omega, k)=-k^{2} \varepsilon_{0} \chi_{e} \phi_{\mathrm{PF}}(\omega, k)-\chi_{e} \delta \rho_{e}(\omega, k), \\
& \delta \rho_{e}=-k^{2} \varepsilon_{0}\left[\chi_{e} /\left(1+\chi_{e}\right)\right] \phi_{\mathrm{PF}}, \\
& \phi_{E}(t, x)=-\left[\chi_{e} /\left(1+\chi_{e}\right)\right] \phi_{\mathrm{PF}}(t, x) .
\end{aligned}
$$

The result for the total effective potential for the electrons, i.e., $\phi_{\text {total }}=\left(\phi_{\mathrm{PF}}+\phi_{E}\right)$, is immediately as follows:

$$
\left(\phi_{\mathrm{PF}}+\phi_{E}\right)=\left[1-\chi_{e} /\left(1+\chi_{e}\right)\right] \phi_{\mathrm{PF}}=\left[1 /\left(1+\chi_{e}\right)\right] \phi_{\mathrm{PF}} .
$$

Approximating the total potential $\phi_{\text {total }}=\left(\phi_{\mathrm{PF}}+\phi_{E}\right)$ trough with a parabolic one $\phi_{\text {total }} \sim-\left[\phi_{\mathrm{PF}} /\left(1+\chi_{e}\right)\right] \cdot\left[1-(k x)^{2}\right]$ near its minimum, the bounce period (or trapping time) of an electron in this potential becomes $\tau_{B}=2 \pi \omega_{B}^{-1}=2 \pi \omega_{p}^{-1}[(1$ $\left.\left.+\chi_{e}\right) / k \lambda_{D} a\right]^{1 / 2}$, where $a=(e / m) k \phi_{\mathrm{PF}} / \omega_{p} v_{\mathrm{Te}}$ is the normalized ponderomotive drive amplitude, $\chi_{e}$ is electron susceptibility, $e$ and $m$ are electron charge and mass, $k$ is the drive wavenumber, $\phi_{\mathrm{PF}}$ is the amplitude of the ponderomotive drive potential, and $\omega_{p}$ and $v_{\mathrm{Te}}$ are plasma frequency and electron thermal velocity, respectively.

Note that for a resonant electron plasma situation such as the Langmuir electron plasma resonance with low loss, $1+\chi_{e}$ is very small, being about $\operatorname{Im}\left(\chi_{e}\right)$, and the bounce period is determined by the very high field of a high- $Q$ resonance. However, for the response at the low frequencies of interest here, $\chi_{e}$ is large and complex, so the electric field is almost antiphase to the PF, leaving a relatively small combined field $\left[\sim 1 /\left(1+\chi_{e}\right)\right]$ nearly in quadrature (i.e., differing in phase by nearly $\pm \pi / 2$ ) with the two large fields (electric and ponderomotive). This behavior can be clearly seen in the early stages of Fig. 3(c).

\section{APPENDIX B: ALLIS-JOHNSTON DENSITY FORMULA}

Allis ${ }^{31}$ suggested a procedure for creating a BGK-type state where one conceptually begins with a known uniform distribution function $f(v)$ in the laboratory with no variation in potential. In a frame moving at the chosen phase velocity $v_{\phi}$, the (nonrelativistic) velocity distribution function under (Galilean) transformation is $f\left(v_{\phi}+u\right)$, where $u$ is the velocity relative to $v_{\phi}$. Assuming that a potential is slowly induced (one could plausibly suppose that this might be done by an appropriate PF), the free-particle velocities could be well approximated by including the effect of the potential adiabatically.

Denoting the original relative velocity at zero potential $(\psi=0)$ by $w$, so that one initially has $f\left(v_{\phi}+w\right)$, so that the original energy (normalized to the particle mass) would be $w^{2} / 2$, then, as the potential $\psi$ is slowly turned up to the proposed value, a particular piece of the velocity distribution function that had the value is assumed to have moved to a new value $u$ which is assumed to be now given by the adiabatic value $u=\operatorname{sgn}(w)\left(w^{2}-2 \psi\right)^{1 / 2}$. For later use it is worth noting that from this one has, of course, the useful inverse result that $w=\operatorname{sgn}(u)\left(u^{2}+2 \psi\right)^{1 / 2}$. The adiabatic distribution is now as follows:

$$
f\left[v_{\phi}+w(u, \psi)\right]=f\left[v_{\phi}+\operatorname{sgn}(u)\left(u^{2}+2 \psi\right)^{1 / 2}\right] .
$$

The convenient potential reference points are the potential maxima, between which particles may be trapped. (Only simple systems are considered, i.e., ones which have no other maxima between the ones at the basic spatial period.) The most convenient maximum potential value $\psi_{\max }$ to take at the maxima is zero, so that in the equivalent stationary system, all free particles necessarily have their total energy greater than zero and trapped particles necessarily have their total energy less than zero. The other useful characteristic value for $\psi$ is $\psi_{\text {min }}$, the lowest potential value at the minimum values interlaced with the maxima. Since all the particles, trapped or free, pass by a potential minimum (one if trapped) or by many (if free), this minimum value $\psi_{\min }$ is also a convenient reference value for all particles, to indicate limit behavior, since as all particles pass through $\psi_{\text {min }}$, their mass normalized kinetic energy $\left(u^{2} / 2\right)$ in the wave velocity frame is necessarily a maximum there, with $u= \pm\left[2\left(E+\left|\psi_{\min }\right|\right)\right]^{1 / 2}$. 
In general, particles with total energy $E$ have relative velocity $u= \pm[2(E-\psi)]^{1 / 2}$ and if these particles pass by the potential maxima (as all free particles do) their relative velocity magnitude is least there, where $\psi=0$, so that at the potential maxima $u= \pm(2 E)^{1 / 2}$. For free particles $(E>0)$ one has $u^{2}$ $\geq-2 \psi>0$, and there is thus a gap in the free-particle velocity distribution function of $\pm|-2 \psi|^{1 / 2}$ around $v_{\phi}$, within which one may find trapped particles.

Allis considered an initially Maxwellian distribution in the laboratory frame, here written as $\exp \left(-v^{2} / 2\right)$, since the normalizing velocity is here taken as $(k T / m)^{1 / 2}$. In the wave phase velocity $\left(v=v_{\phi}\right)$ frame, with relative velocity $w$, one has $\exp \left[-\left(v_{\phi}+w\right)^{2} / 2\right]$. The Allis procedure for the free particles with nonzero $\psi$ is to replace $w$ by $u$, the adiabatic value obtained if the potentially were raised very slowly, to give a distribution function proportional to $\exp \left\{-\left[v_{\phi}+w(u, \psi)\right]^{2} / 2\right\}$. The Allis analysis thus began with the following expression for $f_{\text {free }}(u)$, in which the distribution function density for a Maxwellian, $\exp \left[-\left(v_{\phi}+w\right)^{2} / 2\right]$, is to be assigned to a new velocity $v=v_{\phi}+u$, as indicated below,

$$
\begin{aligned}
& f_{\text {free }}\left(v_{\phi}, u, \psi\right) \sim \exp \left\{-\left[v_{\phi}+w(u, \psi)\right]^{2} / 2\right\}, \\
& f_{\text {free }}\left(v_{\phi}, u, \psi\right) \sim \exp \left(-\left\{v_{\phi}+\operatorname{sgn}(u)\left[\left(u^{2}+2 \psi\right)\right]^{1 / 2}\right\}^{2} / 2\right) .
\end{aligned}
$$

This adiabatic free-particle adiabatic result is also the one given by Eliasson and Shukla ${ }^{30}$ among others. As remarked above, for nonzero $\psi$ the result is a gap in the freeparticle velocity distribution function of $\pm|-2 \psi|^{1 / 2}$ around $v_{\phi}$, and it is there that one may find trapped particles.

No particular value is indicated here for the normalizing constant factor which is needed so the total number of electrons is conserved. (For the uniform Maxwellian with no potential gradient one has the usual Maxwellian value $C_{\langle\psi=0\rangle}=(2 \pi)^{-1 / 2}$, and if there were only free particles the Liouville theorem would indicate the correction for the spatially varying velocity would be simply $w / u$.) Particle trapping is assumed and this is to be accounted for in the end, by integrating over a spatial period of $\psi$, to obtain a normalizing constant that can be denoted by $C_{\langle\psi\rangle}$. This numerical correction is not discussed here, so all that is required here is the form of the distribution function, and the symbol used is simply the $\sim$ symbol.

Turning now to the trapped particles, when the steadystate BGK approximation applies, as many particles are going in one direction as are going in the opposite direction, the trapped-particle distribution function must then be even in $u$, and thus it must be even in $w(u, \psi)$, which is of course not true for the shifted Maxwellian. However Allis noted that, for an original Maxwellian distribution, the expression just given for the free particles could also be rewritten in a form for which the parts which are explicitly separated into two parts which are respectively even and odd in $w(u, \psi)$, as follows:

$$
\begin{aligned}
f_{\text {free }}[w(u, \psi)] \sim & \exp \left\{-\left[v_{\phi}+w(u, \psi)\right]^{2} / 2\right\} \\
\sim & \left.\exp \left[-v_{\phi}^{2} / 2-w(u, \psi)\right]^{2} / 2\right\} \\
& \times\left\{\cosh \left[v_{\phi} w(u, \psi)\right]-\sinh \left[v_{\phi} w(u, \psi)\right]\right\} .
\end{aligned}
$$

Allis proposed simply dropping this odd second term (on the grounds that mixing would tend to reduce it to zero) and thus obtained the following functional form for the trapped particles which could plausibly be made from a Maxwellian, namely, the following expression:

$$
\begin{aligned}
f_{\text {trapped }}\left[v_{\phi}, w(u, \psi)\right] \sim & \exp \left[-v_{\phi}^{2} / 2-w(u, \psi)^{2} / 2\right] \\
& \times\left\{\cosh \left[v_{\phi} w(u, \psi)\right] .\right.
\end{aligned}
$$

Because of the choice of potential used here there are no difficulties of the kind encountered by Allis due to discontinuities across square roots of $w^{2}$ and the like. There is, however, another apparent difficulty for the trapped particles and that is the fact that, if $w^{2} / 2$ is negative real, as required for trapped particles, then $w$ itself must be pure imaginary, so the argument of $\cosh \left[v_{\phi} w(u, \psi)\right]$ is also pure imaginary. Remarkably enough this presents no formal problem, since (as is well known) for real $z, \cosh (i z)$ is simply $\cos (z)$ which is also real and even in $z$. More particularly, we have a new form for $\cosh \left[v_{\phi} w(u, \psi)\right]$ :

$$
\begin{aligned}
\cosh \left[v_{\phi} w(u, \psi)\right] & =\cos \left[i v_{\phi} w(u, \psi)\right] \\
& =\cos \left[\operatorname{sgn}(u) v_{\phi}\left(-2 \psi-u^{2}\right)^{1 / 2}\right] .
\end{aligned}
$$

Hence for the trapped particles with negative energy, the corrected Allis formula becomes the real function as given next:

$$
\begin{gathered}
f_{\text {trapped }}\left[v_{\phi},(-2 \psi)^{1 / 2} \geq u \geq-(-2 \psi)^{1 / 2}, \psi\right] \\
\sim \exp \left[-v_{\phi}^{2} / 2+\left(-2 \psi-u^{2}\right) / 2\right] \\
\times \cos \left[\operatorname{sgn}(u) v_{\phi}\left(-2 \psi-u^{2}\right)^{1 / 2}\right] .
\end{gathered}
$$

This trapped-particle distribution function, being the Allis concept as modified by Johnston, is what is here referred to as the AJ distribution. It is the result of extending the original Maxwellian distribution into the complex plane (i.e., to imaginary velocities). However, given that the present application is not a Laplace transform or Fourier transform calculation, where analytic continuation is often the rule, the justification for the application of this form is not at all clear.

Before discussing how one might employ this formulation, it is worth recalling some fundamentals of trapping. Although the free-particle distribution evolution can be adiabatic, essentially tracking the quasistationary orbits in phase space, the trapped-particle distribution cannot be really adiabatic, since trapping is inherently and essentially a nonadiabatic process. If one defines instantaneous pseudoseparatrices (i.e., defined as if the waves, which are actually slowly varying in the wave frame, were not evolving at all so such pseudoseparatrices could be defined), it is clear that, as the wave is slowly growing, particles become trapped by crossing these pseudoseparatrices in the vicinity of their $(x, v) X$ points, at zero energy, in an essentially nonadiabatic process which can thus drastically separate once close phase-space neighbors in their future dynamics. Also the particles which are captured into the trapping population are necessarily lost from the free-particle population, presumably originally from the free-particle distribution near the separatrices. This microscopic population-transfer aspect is completely ignored here, as is usual in most models of this kind. (Whatever the trapped particle distribution function has been hypothesized 
has, in general, been simply added to the undepleted freeparticle distribution function and then overall particle conservation enforced simply by overall numerical integration over space and renormalization.)

The modified Allis distribution would work if one had a plausible suggestion for the original distribution function in energy to use for the trapped particles, i.e., the equivalent for the Maxwellian that was used for the free particles. To do this one might well have to involve the entire history of the drive. Since the particles come through the separatrix, where $E \sim 0$, one might use the $E=0$, but that would give a flat density over the entire trapped region, which is nothing like the valley centered on $\psi_{\min }$ that is actually observed. Another possibility is to imagine the trapping process to be somewhat like a leak through a barrier, in which case the Maxwellian exponential might be a plausible guess. In the event, it was decided to simply try the formula to see if it worked. An interesting feature of this particular distribution compared with others is that, apart from the overall number conservation, there are no adjustable parameters.

Although the original Allis notation has been followed (more or less) to this point, it is now more convenient to rewrite the results for the free and trapped particles using the original total energy $E\left(=w^{2} / 2\right)$ in the wave frame.

$$
F_{\mathrm{AJ} \pm}(\text { free, } E \geq 0, \psi) \sim \exp \left(-\left[v_{\phi} \pm[2(E-\psi)]^{1 / 2}\right]^{2} / 2\right) .
$$

One actually plots this as a function of $v$ by choosing $E$ $\geq 0$ then calculating from the chosen value of $E$ and the local value for $\psi$ the value for $F_{\mathrm{AJ} \pm}$ (free, $\left.E \geq 0, \psi\right)$ and for $v_{ \pm}$ $=v_{\phi} \pm[2(E-\psi)]^{1 / 2}$ and then plotting them against each other.

For the trapped particles we have the following result:

$$
\begin{aligned}
& F_{\text {AJ }}(\text { trapped, } \psi<E<0, \psi) \\
& \quad \sim \exp \left[-v_{\phi}^{2} / 2+(E-\psi)\right] \cos \left\{v_{\phi}[2(E-\psi)]^{1 / 2}\right\} .
\end{aligned}
$$

The function of $v$ for the trapped particles is now plotted as by choosing $E<0$ then proceeding as for the free particles but using $F_{\mathrm{AJ}}$ (trapped, $\left.\psi<E<0, \psi\right)$ ). It is worth noting that for the trapped-particle AJ form, at $v_{\phi}\left(\psi=\psi_{\min }\right)$ the trapped particle density value is a minimum only for $v_{\phi}>1$ [i.e., only for phase velocity greater than the thermal velocity $\left(k_{B} T / m\right)^{1 / 2}$ which is also the inflection point for the Maxwellian]. However, in the cases of interest for KEEN waves this was always true for the cases considered.

At the velocity separatrices (the values dividing free particles from trapped particles) one has $E=0$. Where the potential has a value $\psi$ in the wave, the two values thus obtained for $v_{ \pm}$for $E=0$ [i.e., $v_{ \pm}=v_{\phi} \pm(-\psi)^{1 / 2}$ ] give the velocity values corresponding to the separatrices. Note that at the separatrices there are generally discontinuities in the distribution function between the trapped and free-particle values, except at $\psi=0$, where both formulas give $\exp \left(-v_{\phi}^{2} / 2\right)$. Recall

$$
\begin{aligned}
F_{\mathrm{AJ} \pm}(\text { free }, E=0, \psi) \sim & \exp \left(-\left\{v_{\phi} \pm[2(-\psi)]^{1 / 2}\right\}^{2} / 2\right) \\
\sim & \left.\exp \left[-v_{\phi}^{2} / 2-\psi \pm v_{\phi}(-\psi)^{1 / 2}\right]\right\}, \\
F_{\mathrm{AJ}}(\text { trapped, } E=0, \psi) \sim & \exp \left(-v_{\phi}^{2} / 2-\psi\right) \\
& \times \cos \left\{v_{\phi}[2(-\psi)]^{1 / 2}\right\} .
\end{aligned}
$$

A final point to note is that when $v_{\phi},[2(-\psi)]^{1 / 2}$ exceeds $\pi / 2$, i.e., when the potential well is too deep, the cosine function passes through zero, and, since negative values for a distribution function are meaningless, some special measures (such as truncation) would be required.

The procedure actually used to obtain the final selfconsistent BGK-like result was to assume the initial values for $\psi$ and to iterate this and the corresponding distribution function (including spatial integration and renormalization so that the total charge is conserved) to an equilibrium result. (This procedure may sometimes fail in that it does not always converge to a nontrivial equilibrium in the sense that $\psi$ values may iterate toward zero in some cases.) When a satisfactory equilibrium result is obtained with usefully large $\psi$, this self-consistent BGK distribution function result is then used as an initial condition for the Vlasov code to verify its stability. (While the existence of a BGK equilibrium arrived at in this nondynamic iteration does not automatically guarantee its survivability in a dynamic Vlasov-Poisson simulation, in practice, when the iteration procedure gives nonzero BGK results, they survive well in the subsequent simulation.) As shown by the data presented in Fig. 17 the AJ result can work quite well, at least sometimes. (Because this is a side issue for the work of this paper, the matter was not pursued further. However, it might be interesting in other contexts to see how well this AJ formulation performs over a range of drive strengths and phase velocities.)

${ }^{1}$ D. S. Montgomery, R. J. Focia, H. A. Rose, D. A. Russell, J. A. Cobble, J. C. Fernández, and R. P. Johnson, Phys. Rev. Lett. 87, 155001 (2001).

${ }^{2}$ D. S. Montgomery, J. A. Cobble, J. C. Fernández, R. J. Focia, R. P. Johnson, N. Renard-LeGalloudec, H. A. Rose, D. A. Russell, H. A. Rose, and D. A. Russell, Phys. Plasmas 9, 2311 (2002).

${ }^{3}$ H. A. Rose and D. A. Russell, Phys. Plasmas 8, 4784 (2001).

${ }^{4}$ K. Watanabe and T. Taniuti, J. Phys. Soc. Jpn. 43, 1819 (1977).

${ }^{5}$ S. P. Gary and R. L. Tokar, Phys. Fluids 28, 2439 (1985).

${ }^{6}$ H. Schamel, Phys. Scr. 20, 336 (1979).

${ }^{7}$ V. P. Krapchev and A. K. Ram, Phys. Rev. A 22, 1229 (1980).

${ }^{8}$ D. J. Strozzi, E. A. Williams, A. B. Langdon, and A. Bers, Phys. Plasmas 14, 013104 (2007).

${ }^{9}$ R. Pottelette, R. E. Ergun, R. A. Treumann, M. Berthomier, C. W. Carlson, J. P. McFadden, and I. Roth, Geophys. Res. Lett. 26, 2629, DOI:10.1029/ 1999GL900462 (1999).

${ }^{10}$ P. K. Shukla, M. A. Hellberg, and L. Stenflo, J. Atmos. Sol.-Terr. Phys. 65, 355 (2003).

${ }^{11}$ I. Bernstein, J. M. Greene, and M. D. Kruskal, Phys. Rev. 108, 546 (1957).

${ }^{12}$ H. Schamel, Phys. Plasmas 7, 4831 (2000), and references therein.

${ }^{13}$ J. P. Holloway and J. J. Dorning, Phys. Lett. A 138, 279 (1989).

${ }^{14}$ J. P. Holloway and J. J. Dorning, Phys. Rev. A 44, 3856 (1991).

${ }^{15}$ M. Buchanan and J. J. Dorning, Phys. Rev. Lett. 70, 3732 (1993); Phys. Rev. E 50, 1465 (1994) c15 c15a c15b c15c 52, 3015 (1995).

${ }^{16}$ C. Lancellotti and J. J. Dorning, Phys. Rev. Lett. 81, 5137 (1998).

${ }^{17}$ C.-H. Lin, J. K. Chao, and C. Z. Cheng, Phys. Plasmas 2, 4195 (1995).

${ }^{18}$ G. Manfredi, Phys. Rev. Lett. 79, 2815 (1997).

${ }^{19}$ M. V. Medvedev, P. H. Diamond, M. N. Rosenbluth, and V. I. Shevchenko, Phys. Rev. Lett. 81, 5824 (1998).

${ }^{20}$ M. Brunetti, F. Califano, and F. Pegoraro, Phys. Rev. E 62, 4109 (2000).

${ }^{21}$ G. Vetoulis and M. Oppenheim, Phys. Rev. Lett. 86, 1235 (2001).

${ }^{22}$ D. L. Newman, M. V. Goldman, M. Spector, and F. Perez, Phys. Rev. Lett. 86, 1239 (2001)

${ }^{23}$ B. Eliasson and P. K. Shukla, Phys. Rep. 422, 225 (2006).

${ }^{24}$ K. Saeki, P. Michelsen, H. L. Pecseli, and J. J. Rasmussen, Phys. Rev. Lett. 42, 501 (1979).

${ }^{25}$ F. Valentini, T. M. O'Neil, and D. H. E. Dubin, Phys. Plasmas 13, 052303 (2006).

${ }^{26}$ T.W. Johnston, B. Afeyan, P. Bertrand, and A. Ghizzo, Paper QP1 136, 
Bull. Am. Phys. Soc. 46(8), 282 (2001); 47(9), 277 (2002); T.W. Johnston and B. Afeyan, Paper CP1 38, ibid. 48(7), 067 (2003); B. Afeyan, V. Savchenko, K. Won, and T.W. Johnston, Paper CP1 39, ibid. 48(7), 068 (2003); T.W. Johnston, Y. Tyshetskiy, and B. Afeyan, Paper BP1 52, ibid. 49(8), 40 (2004); Y. Tyshetskiy, T.W. Johnston, and B. Afeyan, Paper BP1 53, ibid. 49(8), 40 (2004); J. Kline, B. Afeyan, W. Bertsche, N. Kurnit, D. Montgomery, V. Savchenko, and K. Won, Paper BP1 56, ibid. 49(8), 41 (2004); Y. Tyshetskiy, T.W. Johnston, and B. Afeyan, Paper LP1 74, ibid. 50(8), 242 (2005); K. Won, B. Afeyan, V. Savchenko, P. Morrison, and T. W. Johnston, Paper LP1 75, ibid. 50(8), 242 (2005); T.W. Johnston, Y. Tyshetskiy, and B. Afeyan, Paper UP1 118, ibid. 51(7), 288 (2006); V. Savchenko, K. Won, and B. Afeyan, Paper UP1 119, ibid. 51(7), 288 (2006); B. Afeyan and M. Charbonneau-Lefort, Paper GP8 37, ibid. 52(16), 116 (2007).

${ }^{27}$ B. Afeyan, K. Won, V. Savchenko, T. W. Johnston, A. Ghizzo, and P. Bertrand, "Kinetic electrostatic electron nonlinear (KEEN) Waves and their interactions driven by the PF of crossing laser beams," in Third International Conference on Inertial Fusion Sciences and Applications, Monterey, CA, 7-12 September 2003, edited by B. Hammel, D. Meyerhofer, J. Meyer-ter-Vehn, and H. Azechi (American Nuclear Society, LaGrange Park, IL, 2004), Paper No. M034, p. 213.

${ }^{28}$ B. Afeyan, Bull. Am. Phys. Soc. 49, 288 (2004).

${ }^{29}$ C. Z. Cheng and G. Knorr, J. Comput. Phys. 22, 330 (1976).
${ }^{30}$ A. Ghizzo, P. Bertrand, M. Shoucri, T. W. Johnston, E. Fijalkow, and M. R. Feix, J. Comput. Phys. 90, 431 (1990).

${ }^{31}$ R. N. Bracewell, The Fourier Transform and Its Applications (McGrawHill, New York, 1978); also Science 248, 697 (1990); A. Ghizzo, T. Reveille, P. Bertrand, T. W. Johnston, J. Lebas, and M. Shoucri, J. Comput. Phys. 118, 356 (1995). The application here using the Hilbert transform is very simple, but the treatment of much more complicated situations can be found via the Hilbert-Huang analysis, which uses empirical mode decomposition, as set out in Hilbert-Huang Transform and Its Applications, Interdisciplinary Mathematical Sciences Vol. 5, edited by N. E. Huang and S. S. P. Shen (World Scientific, Singapore, 2005).

${ }^{32}$ B. Eliasson and P. K. Shukla, Phys. Rev. E 71, 046402 (2005).

${ }^{33}$ W. P. Allis, in In Honor of Philip M. Morse, edited by H. Feshbach and K. Ingard (MIT, Cambridge, 1969), pp. 21-42.

${ }^{34}$ H. L. Berk, C. E. Nielsen, and K. V. Roberts, Phys. Fluids 13, 980 (1970).

${ }^{35}$ P. Bertrand, A. Ghizzo, M. R. Feix, E. Fijalkow, P. Mineau, and N. D. Suh, and M. Shoucri, Proceedings of the International Workshop on Nonlinear Phenomena in Vlasov Plasmas, Cargèse, France, 11-16 July 1988, edited by F. Doveil (Editions de Physique d'Orsay, Orsay, 1989), p. 109; see also A. Ghizzo, B. Izrar, P. Bertrand, E. Fijalkov, M. R. Feix, and M. Shoucri, Phys. Fluids 31, 72 (1988).

${ }^{36}$ M. Albrecht-Marc, A. Ghizzo, T. W. Johnston, T. Réveillé, D. Del Sarto, and P. Bertrand, Phys. Plasmas 14, 072704 (2007). 